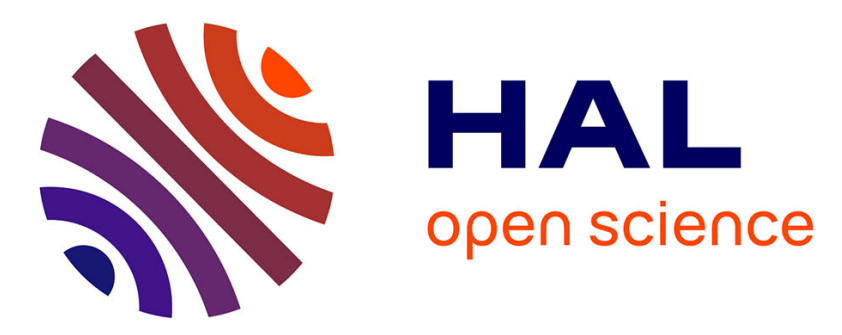

\title{
La santé et sécurité au travail au sein des congrès de la communauté AGRH: retour sur 25 ans de travaux et perspectives futures de recherche
} Tarik Chakor, Emmanuel Abord de Chatillon, Olivier Bachelard

\section{- To cite this version:}

Tarik Chakor, Emmanuel Abord de Chatillon, Olivier Bachelard. La santé et sécurité au travail au sein des congrès de la communauté AGRH : retour sur 25 ans de travaux et perspectives futures de recherche. @GRH, 2015, 15, pp.127 - 165. 10.3917/grh.152.0127 . hal-01393648

\author{
HAL Id: hal-01393648 \\ https://hal.science/hal-01393648
}

Submitted on 7 Nov 2016

HAL is a multi-disciplinary open access archive for the deposit and dissemination of scientific research documents, whether they are published or not. The documents may come from teaching and research institutions in France or abroad, or from public or private research centers.
L'archive ouverte pluridisciplinaire HAL, est destinée au dépôt et à la diffusion de documents scientifiques de niveau recherche, publiés ou non, émanant des établissements d'enseignement et de recherche français ou étrangers, des laboratoires publics ou privés. 


\title{
"LA SANTE ET SECURITE AU TRAVAIL AU SEIN DES CONGRES DE LA COMMUNAUTE AGRH: RETOUR SUR 25 ANS DE TRAVAUX ET PERSPECTIVES FUTURES DE RECHERCHE"
}

(Version n-1 quasi-définitive de l'article sélectionné et publié dans le numéro spécial Meilleurs papiers AGRH 2015 de 1'@GRH)

\author{
Tarik Chakor \\ Maître de conférences - Université Savoie Mont Blanc \\ IREGE EA 2426 \\ tarik.chakor@univ-smb.fr
}

Emmanuel Abord de Chatillon

Professeur des Universités - Chaire Management et Santé au Travail

CERAG, IAE de Grenoble

abord@iae-grenoble.fr

\section{Olivier Bachelard}

Enseignant-chercheur - EM Lyon

obachelard@em-lyon.com

\section{Pour citer cet article :}

Chakor T., Abord de Chatillon E., Bachelard O. (2015), " La Santé et Sécurité au Travail au sein des congrès de la communauté AGRH : retour sur 25 ans de travaux et perspectives futures de recherche", @GRH,2015/2,p. 127-165.

L'objectif de notre article est de présenter un état des lieux de la recherche en Santé et Sécurité au Travail (SST) au sein des congrès de la communauté AGRH, depuis leur approche marginale et indirecte lors des premiers événements (Bordeaux, 1990 ; Cergy, 1991) jusqu'à l'hétérogénéité actuelle des angles d'appréhension et niveaux d'analyse de cette thématique, en nous basant sur une recherche bibliographique exhaustive réalisée à partir des Actes de Colloque de l'AGRH. Cette méta-analyse doit ainsi permettre de mettre en relief le fait que la communauté AGRH a été tout d'abord relativement discrète sur ces questions, voire totalement absente, la SST constituant une sorte de « point aveugle » des travaux en GRH.

L'intensification du travail et les limites des approches strictement techniques du management des RH ont notamment permis de populariser cette thématique, la SST devenant le symbole des limites et des insuffisances de la GRH. On observe ainsi la constitution d'un corpus théorique de plus en plus étoffé, abordant l'émergence de nouveaux concepts (tels que le stress, le burnout, le harcèlement, les RPS, plus récemment le bien-être), les pratiques et acteurs de la SST, les sources et facteurs de troubles, la multiplicité des approches disciplinaires, ainsi que la mesure de ces différents phénomènes.

\section{Mots-clés}

Santé, sécurité, travail, AGRH 


\section{Introduction}

L'Association française de Gestion des Ressources Humaines (AGRH) a organisé son premier congrès les 27 et 28 Novembre 1990, événement intitulé «Perspectives de l'entreprise et recherche en G.R.H., les défis des années 1990 ».

Lors de son allocution d'ouverture, le premier président de l'association, Jacques Igalens, a cité un article issu d'un numéro de Business Week, soulignant le manque d'intérêt des dirigeants d'entreprise pour la production scientifique des business-schools nord-américaines, cette production étant concentrée sur un nombre restreint de sujets ne correspondant nullement à leurs besoins (Igalens, 1990). La volonté affichée était alors de développer au sein de l'AGRH des recherches alliant qualité de la démarche scientifique et pertinence des orientations retenues. C'est ainsi que les huit premières commissions traitaient de sujets aussi divers que l'évolution de la fonction, l'évolution des hommes de la fonction, le développement de l'organisation, l'appréciation et la rémunération, les autres regards sur la $\mathrm{GRH}$, la gestion des carrières et des compétences, l'emploi et la gestion prévisionnelle, et enfin le développement personnel et l'implication. D'autre part, l'allocution d'ouverture du président de l'Association Nationale des Directeurs et Cadres de la fonction Personnel (ANDCP, ex-ANDRH), Robert Bosquet, a souligné quant à elle le rôle-clé du DRH, notamment «pour ce qui concerne la satisfaction, la motivation et l'implication des hommes » (Bosquet, 1990, p 9). Ce dernier considérait alors, déjà, que l'efficacité collective était un défi dont les acteurs de l'organisation devaient prendre conscience, citant Jean-Léon Donnadieu : l'entreprise se fait «avec, par et pour les hommes». Enfin, Robert Bosquet a émis certaines attentes vis-à-vis des travaux des chercheurs en GRH : éclairer la complexité des défis que les praticiens ont à résoudre; les aider à comprendre les fondements de la vie humaine en entreprise, les aspirations des hommes, sans perdre de vue les interlocuteurs de terrain, afin de faire progresser les conditions de vie de millions de salariés, ainsi que la performance économique (ibid., 1990).

Cette double allocution d'ouverture résume, à elle seule, la place de la thématique « Santé et Sécurité au Travail» (SST) au sein de la communauté AGRH durant la période 1990-2000 : une thématique sous-jacente, abordée indirectement, au détour de concepts plus ou moins proches tels que le développement personnel, l'implication, la satisfaction ou les conditions de vie. Progressivement, au détour des années 2000, la place de la SST va s'accroître, jusqu'à constituer une thématique centrale de recherche au sein de l'AGRH.

C'est ainsi que cet article se propose de retracer, au fil des 25 années d'existence des congrès de l'AGRH, les différents travaux, recherches et analyses portant sur la SST. Nous avons ainsi procédé à une recherche bibliographique exhaustive, réalisée à partir des Actes de Colloque de l'AGRH disponibles sur le site de l'association.

D'un point de vue méthodologique, nous avons identifié dans un premier temps tous les textes paraissant avoir un lien avec la problématique SST, même lointain. Après une première lecture, certains ont été mobilisés soit pour leur capacité à rendre compte de l'état des recherches en Gestion des Ressources Humaines à un moment donné, soit pour leur contribution directe au champ de la SST. Ce travail initial a été doublement effectué par les auteurs, ce qui a permis d'ajouter et de retrancher après discussion une dizaine de textes. L'ensemble des textes mentionnés figurent d'ailleurs dans la bibliographie initiale alors que le tableau des auteurs ayant contribué à la thématique ne reprend que les textes que nous considérons appartenir directement au champ de la Santé et Sécurité au Travail. S'est donc 
posée la question du contour de ce champ. Si un ensemble de travaux ne posent pas d'interrogations car mentionnant explicitement un travail sur une pathologie, sur le bien-être, ou une catégorie d'acteurs directement liée à la question (par ex. les médecins du travail), d'autres ont fait l'objet de débats. C'est ainsi que les travaux portant sur l'engagement ont été écartés (sauf si cette question était directement associée à une problématique de SST), mais qu'en revanche ceux concernant l'équilibre personnel / professionnel ont été retenus du fait de leur lien étroit avec la question du bien-être au travail.

À partir de cette base ainsi constituée, une nouvelle lecture des textes a permis d'organiser des phases et des logiques distinctes qui ont donné lieu à débats au sein de l'équipe de recherche puis à une mise en forme structurée.

Cet état des lieux historique permet de distinguer deux périodes distinctes, et l'amorce d'une troisième période :

- la première période, du congrès de Bordeaux en 1990 à celui de Lyon en 1999, est une période où la SST était abordée de manière marginale ou indirecte, voire totalement ignorée des travaux de recherche de la communauté AGRH, même si l'émergence de premiers travaux abordant directement cette thématique, couplée à ce que nous nommerons «l'exception québécoise », permettent d'atténuer ce sentiment;

- la deuxième période, du congrès de Paris en 2000 à celui de Chester en 2014, a permis d'observer la constitution progressive d'un corpus théorique de plus en plus fourni, corpus mêlant des travaux autour de concepts émergents en SST, des pratiques et des acteurs de ce nouveau champ de recherche, une pluralité d'approches disciplinaires, ainsi que la volonté affichée de mesurer la SST ;

- la troisième période semble s'amorcer aujourd'hui et nous permettra de conclure ce travail. Constatant la place toujours plus importante des thématiques de SST sur les scènes économiques, sociales, juridiques et politico-médiatiques d'une part, et à la multiplication des initiatives, notamment scientifiques et institutionnelles, d'autre part, elle permet de décrire ce champ de recherche comme symbolique d'une problématique de Gestion des Ressources Humaines qui repose sur un ensemble de connaissances issues des disciplines connexes pour développer progressivement un corps de connaissances autonome.

\section{De 1990 à 2000 : une approche marginale et indirecte de la SST par la communauté AGRH - La période d'insouciance}

Les premiers débats au sein de l'AGRH portaient principalement sur la notion même de "gestion des ressources humaines », appellation nouvellement consacrée, au détriment du désuet «gestion du personnel ». Ainsi, les débats de l'époque semblent s'être naturellement orientés autour de la « fonction $\mathrm{RH}$ », et plus largement autour de l'approche fonctionnelle et mécaniste de l'entreprise (1.1.). Cette approche concourait à un certain flou autour de la place de l'être humain dans l'entreprise, de sa santé et de sa sécurité, éléments appréhendés au détour de concepts et notions connexes à la SST (1.2.). Cette période a toutefois vu émerger des premiers travaux français articulant GRH et SST (1.3.), et des travaux québécois abordant directement et de manière approfondie cette thématique (1.4.). 


\subsection{Une approche fonctionnelle et mécaniste de la Gestion des Ressources Humaines : la SST comme point aveugle des travaux en GRH}

La dimension humaine des entreprises a fait partie intégrante des enjeux et défis de l'AGRH dès la double allocution d'ouverture, mais essentiellement au prisme de l'entreprise et de son organisation fonctionnelle. L'étude du contenu du premier congrès, ayant eu lieu à Bordeaux en 1990, est à ce titre très instructive : tandis que la première séance plénière était intitulée "Recherche sur la fonction et fonctions de la recherche », l'essentiel des communications se positionnait à un niveau que l'on pourrait qualifier de macro-économique. Ainsi, la gestion stratégique des ressources humaines, le développement de nouvelles structures et morphologies d'entreprises, le cas des entreprises-réseau, les entreprises décentralisées, les formes alternatives d'emploi, la flexibilité croissante et les politiques d'internalisation ou externalisation du travail semblent se trouver au cœur des réflexions en GRH (Weiss, 1990). $\mathrm{La}$ "gestion du facteur humain» est appréhendée comme devant être cohérente avec la stratégie de l'entreprise (ibid., 1990). L'expression "politique de personnel » est énoncée, tout en étant conservée dans un certain flou sémantique, la dimension stratégique et, plus largement, économique de l'entreprise prenant le pas sur la dimension strictement humaine.

Ainsi, la communication de Louis Gosselin (1990), intitulée «La fonction Ressources Humaines : modèle et typologie » est focalisée sur la manière dont la fonction RH se caractérisait et a évolué au sein de l'entreprise. La communication d'Alain Roger (1990), intitulée "De la gestion du personnel à la gestion des ressources humaines », éclaire la manière dont ont évolué les termes utilisés pour décrire la fonction "personnel» et « ressources humaines»: l'auteur souligne l'importance accordée à la formation, à la communication et à la gestion des carrières, au-delà de la traditionnelle gestion du personnel. Ici, le terme " ressources » semble privilégié au terme " humaines », les entreprises ayant un souci croissant d'optimisation de leurs ressources, dans leur globalité.

L'approche fonctionnelle et mécaniste de la GRH se vérifie également au sein de nombreuses présentations du congrès de 1990: «La place de la fonction Ressources Humaines en Europe : similitudes et différences » (Bournois, 1990), soulignant des différences nationales significatives à la veille du marché unique de 1993 ; "La fonction Personnel : évolutions et différenciations » (Brabet et Fenneteau, 1990) et les différences objectives entre organisations ; "Les métiers de la fonction Ressources Humaines » (Duyck, 1990) ; «Le flux de personnels et achats de services » (Noel, 1990). La communication de Christel Beaucourt (1990), intitulée "Pour une gestion sociale ajustée », permet cependant d'atténuer la vision d'une approche strictement fonctionnelle de la GRH, cette dernière énonçant l'idée que les politiques de mobilisation du personnel se heurtent à la complexité de l'être humain, devant ainsi s'adapter à lui et prendre en compte son engagement.

\section{2. "Flou humain " et concepts connexes à la SST}

La place de l'humain dans l'entreprise est essentiellement sous-jacente au sein des travaux présentés lors des premières manifestations de l'AGRH. La "gestion du facteur humain » et des « problèmes humains » (Weiss, 1990) et la mobilisation et la gestion des hommes (Amado et Deumie, 1990) sont appréhendées au prisme de leur dimension économique et de la performance de l'entreprise (Weiss, 1990), le développement des hommes étant intégré dans les objectifs de rentabilité du capital (Bartoli, 1990). 
Au-delà de cette approche de type socio-économique, le contenu de l'activité de travail est abordé, notamment via la commission intitulée "L'évolution des hommes de la fonction » (AGRH, 1990) : ce contenu définit l'homme au travail, les exigences auxquelles il doit faire face, ses stratégies de conciliation, notamment les dimensions de l'activité potentiellement contradictoires (Pouget, 1990). En s'appuyant sur le concept de complexité d'Edgar Morin, Gilles Amado et Claudine Deumie (1990) soulignent le fait que l'individu ne doit pas être appréhendé comme une ressource à exploiter mais plutôt comme un sujet humain en devenir et complexe, aux souffrances spécifiques. Cette dimension spécifique tend à justifier le nécessaire examen des situations de travail de manière approfondie, afin de comprendre les exigences de chaque tâche et articuler personnes et travail.

On observe ainsi la multiplication de concepts connexes à la SST, sans pour autant placer cette thématique au cœur des analyses : caractérisées par l'intensification du travail, les mutations du management, la recherche de qualité totale et l'obsession managériale, les entreprises développent une face d'ombre, allant à l'encontre de leur dimension spirituelle, où l'entreprise peut constituer une instance de développement personnel (Aubert, 1991). Ces « maladies de l'excellence » (stress permanent, décompressions physiques et psychiques, malêtre, angoisse) sont appréhendées comme «le cô̂t de l'excellence», Nicole Aubert présentant sa recherche et son ouvrage du même nom co-écrit avec Vincent de Gaulejac (ibid., 1991). La satisfaction à l'égard des conditions et des caractéristiques de travail (Bernatchez, 1991 ; Poilpot-Rocaboy, 1994 ; Roussel, 1994 ; Berman, 1995 ; Kopel et Sire, 1997 ; Legault et Simard, 1998), l'autonomie des personnels (Delperier, 1990), l'implication des salariés et l'adhésion aux pratiques RH de l'entreprise (Bernard, 1991 ; Tissier-Desbordes \& Thevenet, 1991; Peyrat, 1993; Paillé, 1996; Neveu et Peyrat-Guillard, 1998), les congruences et divergences entre les systèmes de valeur du salarié et de l'entreprise (Neveu, 1991), l'émergence des travaux entre 1980 et 1990 autour de l'engagement au travail et de ses conditions psychologiques (Igalens et Penan, 1991), la mobilisation des personnes (Aktouf, 1991) et enfin le sens du travail (Fiol et Solé, 1993), témoignent de la croissance de ces objets de recherche connexes. L'analyse du vocabulaire de la GRH souligne cependant la prédominance des termes personnel, gestion, formation, expérience, fonction, le terme «humain(e)s " n'arrivant qu'en $6^{\text {ème }}$ position (Duyck, 1991), confirmant l'approche fonctionnelle et mécaniste de la GRH énoncée précédemment.

\section{3. Émergence des premières articulations entre GRH et SST}

Des premiers travaux amorcent toutefois l'articulation « directe » entre GRH et SST : ainsi, la communication de Marc Bartoli (1990), intitulée «Gestion des ressources humaines ou du travail humain ", souligne la réalité tridimensionnelle du travail (mobiles, buts, moyens) et l'importance pour la GRH de passer du travail prescrit à la prescription de l'engagement des personnes, le travail humain devant constituer un objet central et unificateur. Cette conception multidimensionnelle du travail s'appuie notamment sur les travaux d'Yves Clot, consacrée à l'analyse du travail en situations de mutations technologiques chez PSA. Pierre Louart (1990) considère la gestion du réel comme l'un des paradoxes de la GRH : en distinguant réalité physique et réalité psychique, l'auteur souligne la complexité personnelle et les spécificités psychologiques individuelles à mieux gérer par la GRH. Cette idée de complexité du réel est également soulignée par Christophe Vignon (1990) dans son rapport sur le contenu de la Commission «Autres regards sur la GRH»: ainsi, "la gestion doit ouvrir son champ conceptuel. Il est souhaitable d'abandonner les modèles stéréotypés et globalisants au profit d'un savoir qui intègre la complexité du réel »(Vignon, 1990, p 342). 
Au-delà de la question du travail réel, la question de la sécurité au travail a émergé dès le début des années 90 au sein de la communauté AGRH : tout d'abord liée aux problèmes de conciliation entre la mobilité de métier et la recherche de stabilité et sécurité par le salarié (Sire, 1990), elle s'est ensuite orientée vers l'étude du lien entre sécurité du travail et pratiques de gestion. En effet, l'étude du lien entre assurance qualité et occurrence des accidents de travail (Abord de Chatillon, 1992) et l'évaluation du lien entre performance et gestion de la sécurité du travail (Abord de Chatillon, 1996) ont mis en évidence le paradoxe semblant exister entre outillage sécurité accru et performance sécurité amoindrie. Ces études pionnières ont permis de faire émerger une proposition de pilotage de la sécurité du travail fondé sur les représentations des acteurs et des moyens d'action concrets et opérationnels telle que la méthode RECORE (REprésentations, COmportements, REgles) (ibid., 1996 ; Abord de Chatillon, 1997), visant à améliorer la gestion des comportements et des attitudes face au problème de la sécurité du travail. L'idée de philosophie de management de la sécurité apparaît, ainsi que les principes d'engagement et de participation des acteurs, ces parties prenantes devant se transformer en acteurs de la prévention dans la durée (Abord de Chatillon, 1998). Ces méthodes de diagnostics complexes répondent ainsi à des situations accidentelles également complexes, résultant de processus non mécanistes et non linéaires.

On constate enfin, durant cette période, la volonté de proposer une véritable ingénierie des interventions en SST, d'une part autour de la santé psychologique au travail (Neveu, 1996), et d'autre part autour de l'équilibre entre autonomie et contrôle (Abord de Chatillon et Vauclin, 1999), afin d'affronter les "nouveaux maux de cette fin de siècle: stress, dépression, harcèlement et épuisement professionnel » (ibid., 1999, p 22). Le stress constitue l'un des premiers maux psychosociaux ayant été abordé durant les années 90 au sein de l'AGRH : en effet, Alain Roger et Olivier Roques ont analysé les variations de l'intensité du stress perçu aux différentes étapes de carrière des cadres, distinguant le stress d'abandon, du stress de surcharge et du stress d'ambigüité (Roger et Roques, 1994). Ces mêmes auteurs ont étudié les déterminants et les modes de réaction au stress au travail des cadres (Roger et Roques, 1995), avant de s'intéresser aux réactions de stress des salariés face aux transitions de carrière (Roques, 1996).

\subsection{L'exception québécoise : la SST comme objet de recherche à part entière}

Nous nommons ici «exception québécoise » le fait que, contrairement aux travaux des chercheurs français de l'AGRH, ceux des chercheurs québécois se sont très rapidement intéressés à la question de la SST. Ainsi, dès le congrès de 1991 à Cergy, la communication de Jacqueline Dionne-Proulx, de l'Université du Québec à Trois-Rivières, était intitulée « $L a$ promotion de la santé au travail, un outil de gestion ", intervention considérant que cette promotion pouvait permettre de maximiser la compétitivité et la productivité (Dionne-Proulx, 1991). L'auteure se penche sur la structuration des milieux de travail, et la recherche de nouvelles opportunités d'équilibrer les objectifs personnels, sociaux, organisationnels et fonctionnels dans les organisations. Elle propose ainsi une représentation schématique de la promotion de la santé au travail, intégrant les dimensions opérationnelles, les facteurs de contingence, ainsi que les effets individuels et organisationnels (schéma 1, ci-dessous). 


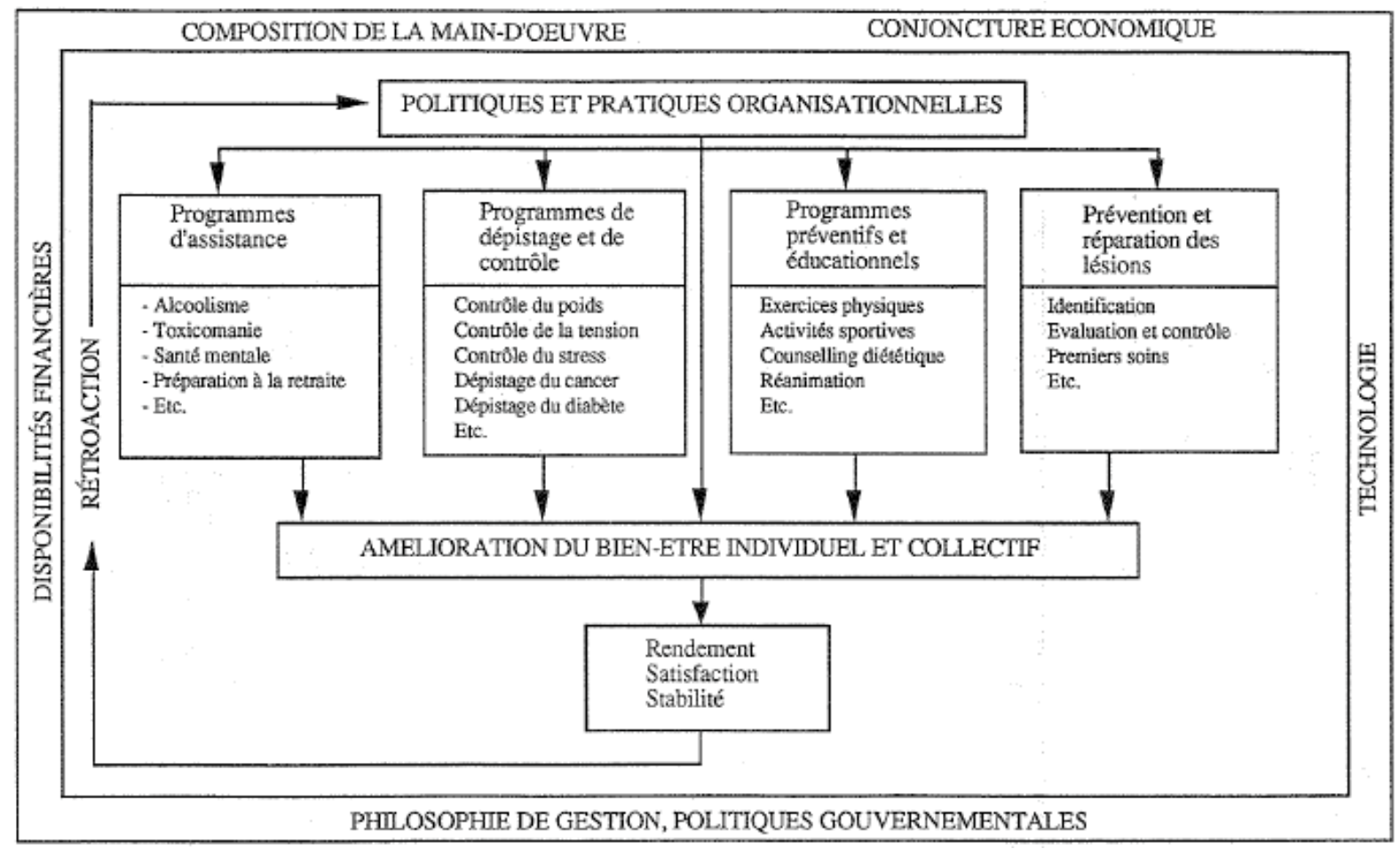

Schéma 1. Représentation schématique du processus de promotion de la santé au travail (extrait de Dionne-Proulx J. (1991), " La promotion de la santé au travail », Actes du second colloque de l'AGRH, Cergy, 14-15 Novembre 1991, p. 504)

La promotion de la santé au travail n'était pas l'unique porte d'entrée des études québécoises en SST : 1'analyse du malaise des ingénieurs syndiqués, associé au manque d'autonomie, de reconnaissance et de participation (Wils et Guérin, 1993), mais surtout la question de l'équilibre travail - famille, ou vie professionnelle - vie personnelle, ont constitué d'autres voies d'appréhension de la thématique SST. L'équilibre travail - famille est ainsi apparu comme un enjeu organisationnel, les évolutions démographiques et sociologiques ayant fait apparaître de nouveaux besoins chez les employés (St-Onge, Guérin, Trottier, Simard et Haines, 1993), certaines entreprises, notamment publiques, de grande taille et fortement syndiquées, ayant mis en place différentes pratiques telles des congés adaptés, des avantages sociaux et des aménagements du travail (Guerin, St-Onge, Wils, Haines, Trottier et Simard, 1994). Enfin, les travaux de Dominique Bouteiller autour des liens entre gestion participative et SST ont pu permettre d'éclairer le fait que le participatif, en tant que mode de prise en charge des problèmes SST, peut avoir un effet structurant, même si limité, sur le système d'action patronal, voire bouleverser la structure organisationnelle (Bouteiller, 1994).

L'explication de cette " exception québécoise » tient aussi bien au caractère plus pragmatique des chercheurs québécois, mais aussi à une plus grande porosité des disciplines scientifiques des sciences du travail en Amérique du Nord, ainsi qu'au soutien financier dont ont pu bénéficier nombre de travaux en la matière de la part des organismes publics et semi-publics.

Cette première partie nous a permis de mettre en évidence le fait, que durant la période 1990-2000, les premiers débats au sein de l'AGRH portaient principalement sur le contenu de la fonction RH, l'être humain dans l'entreprise et la SST étant appréhendés via des concepts et notions connexes, bien que certains ont progressivement émergé en France et plus 
largement au Québec. La deuxième partie nous permet de mettre en évidence la place accrue de la thématique SST au sein de la communauté AGRH, avec l'étude de concepts, pratiques, acteurs, sources et outils de mesures spécifiques à ce champ de recherche.

\section{De 2000 à nos jours : concepts, pratiques et acteurs, sources, mesure : la SST dans tous ses états - La prise de conscience}

L'intensification du travail, les limites des approches techniques du management des RH et l'évolution du cadre juridique, depuis notamment les premiers textes de 1991 avec l'obligation de sécurité et de santé (responsabilité civile et pénale), ont favorisé le développement et la popularisation des travaux en SST, ainsi que leur pénétration explicite dans le champ de la Gestion des Ressources Humaines.

À cet égard, il est à noter que le rôle de l'audit social fut tout à fait significatif. En effet, le premier atelier consacré à la SST et au lien avec la GRH a été organisé en 2003, à l'occasion de la $5^{\text {ème }}$ Université de Printemps de l'Institut de l'Audit Social (IAS). La logique de l'audit social a ainsi montré les défaillances de la GRH à intégrer cette dimension SST pour articuler de façon satisfaisante compétitivité et bien-être au travail. Une dynamique de recherche s'est alors développée autour de la communauté de l'audit social. À la suite de cela, la création d'un GRT (Groupe de Recherche Thématique) de l'AGRH a permis de soutenir la production individuelle et collective autour de la SST. La volonté de construire un espace diversifié d'échange des pratiques, de confrontation et de structuration de connaissances a ainsi progressivement vu le jour au sein de l'AGRH. Les premières journées de recherche pluridisciplinaires francophones AGRH de la Santé et Sécurité au Travail (Annecy, 7-8 avril 2005), puis les secondes sur le management de la Santé et de la Sécurité au Travail (Saint Etienne, 26-27 avril 2007) ont également contribué à réunir les chercheurs et les praticiens autour de cette problématique en favorisant son émergence. De cette mise en réseau des chercheurs et des praticiens, un premier ouvrage a regroupé les productions (Abord de Chatillon et Bachelard 2005), puis un second dans la collection AGRH recherche (Abord de Chatillon, Bachelard et Carpentier, 2012). Ce développement s'est traduit par de nouvelles productions scientifiques contribuant à l'émergence de nouveaux concepts (2.1.), de pratiques et d'acteurs spécifiques (2.2.), de sources, facteurs et approches disciplinaires multiples (2.3) et de la volonté de mesurer la SST (2.4).

\subsection{L'émergence de concepts spécifiques en SST}

\section{Le harcèlement}

Dès le congrès de 1998 à Versailles, et parallèlement au succès de l'ouvrage de MarieFrance Hirigoyen (1998) sur cette thématique, le concept de harcèlement professionnel a été abordé par Gwénaëlle Poilpot-Rocaboy, cette dernière considérant que la fonction RH devait être le garant du respect du contrat psychologique relationnel dans l'entreprise, au détour de pratiques proactives et réactives (Poilpot-Rocaboy, 1998). L'auteure poursuit ses réflexions, appréhendant ce phénomène comme une possible pratique de GRH au service de stratégies de croissance externe, amenant les salariés à rompre volontairement, du moins en apparence, leur contrat de travail (Poilpot-Rocaboy, 2002). Le phénomène de harcèlement sera ainsi au cœur de plusieurs communications, liant harcèlement moral et risque organisationnel (Carpentier, 2004 ; Champion et Paturel, 2006), harcèlement psychologique au travail et processus d'intervention (Poilpot-Rocaboy et Winter, 2006), ainsi que comportements de harcèlement moral au travail et ambiguïtés comportementales (Deharo et Point, 2013). Il convient de 
préciser que la loi de modernisation sociale du 17 Janvier 2002, qui définit juridiquement le harcèlement, a assurément favorisé un nombre accru de travaux sur cette thématique.

\section{Le stress et les RPS}

Un changement de sémantique s'opère en quelques années et les travaux initialement focalisés sur le stress mutent progressivement avec l'émergence du concept de risques psychosociaux au travail (RPS) en France. Dans la continuité de ses travaux amorcés à la fin des années 90, Olivier Roques a étudié l'influence du lieu de contrôle sur le stress au travail (Roques, 2000) et réalisé huit études de cas d'entreprises suivies par des médecins du travail, afin de promouvoir la construction d'une méthode d'intervention en matière de prévention du stress professionnel (Roques, 2001). Danielle Picard a, quant à elle, opéré une analyse comparée des travaux anglo-saxons sur le stress professionnel et des recherches de l'école française de psychodynamique du travail, afin de réfléchir aux conciliations possibles à envisager, notamment l'approche de la subjectivité de l'homme au travail, la compréhension de ses stratégies défensives et l'éthique de la parole et de la discussion contradictoire, allant à contre-courant de la tendance dominante individualiste (Picard, 2000). Claire Edey-Gamassou s'est intéressée aux liens entre innovation et stress professionnel, l'innovation pouvant améliorer les conditions de travail et l'efficacité organisationnelle (Edey-Gamassou, 2003). D'autres travaux autour du stress et des RPS ont ainsi progressivement émergé : l'évaluation de la mesure du stress (Cadieux, Roy et Desmarais, 2004 ; Poirot, 2004), l'étude des liens entre stress et violence organisationnelle (El Akremi et Sassi, 2005), le cas du stress des infirmières (Colle et Moisson, 2007), les relations entre stress et cynisme (Roques et Serrano Archimi, 2009) ou entre stress, performance et leadership transformationnel (Molines, 2010), les représentations du stress des dirigeants et leurs implications pour la gestion du stress (Pezé, 2010), le stress des managers territoriaux (Codo, 2010), l'impact des conditions de travail sur le stress perçu (Mansour, 2012 ; Mansour et Commeiras, 2013), les liens entre stress et implication organisationnelle (Carrier Vernhet et Desmarais, 2012), les stratégies de coping et d'ajustement liées au stress (Abbas et Roger, 2013 ; Montargot, Cherkaoui et Peretti, 2013), les compétences politiques et sociales comme remède aux stress des dirigeants (Poirot, 2005) et enfin la généalogie des RPS au travail (Chakor, 2014 ; Chakor, 2015). Le premier ouvrage mentionnant les RPS en France date de 2008 sous le titre " Risques psychosociaux, vraies questions et bonnes réponses " (Grasset, Debout, Rouat et Bachelard, 2008). Cet intérêt pour le stress et les RPS a notamment été permis par la parution de multiples rapports (Gollac \& Bodier, 2011 ; Lachmann, Larose \& Penicaud, 2010 ; Nasse \& Légeron, 2008) d'une part, et par la médiatisation des suicides chez France Télécom ou Renault d'autre part, favorisant l'émergence d'un débat national.

\section{La sécurité au travail}

Les travaux de recherche dans ce domaine se sont renforcés avec l'apparition d'une contrainte légale : le décret du 5 novembre 2001 et une circulaire du 18 avril 2002. L'évaluation des risques obligatoire depuis 1991 (art. L 230-2 du code du Travail) doit être consignée dans un document unique (art. R 230-1). Le risque professionnel est progressivement devenu de ce fait un enjeu juridique majeur avec l'obligation pour l'employeur de préserver la santé et la sécurité des salariés et d'y travailler.

\section{Épuisement professionnel/burnout, pénibilité, souffrance, violence, émotions}


Parallèlement, d'autres concepts de SST sont apparus au fur et à mesure (Bachelard et Paturel, 2004) : l'épuisement professionnel, ou burnout, et leurs liens avec l'agressivité (Neveu, 2006), avec le stress (Moisson et Edey-Gamassou, 2008), avec la violence au travail (Carpentier, 2004 ; Cintas, 2006 ; Cintas et Sprimont, 2011), ou l'employabilité des cadres (Frimousse et al., 2013), le burnout et la déviance organisationnelle (Abord de Chatillon et Neveu, 2009) ou celui des internes en médecine (Cousineau et Langlois, 2014). La pénibilité au travail et sa perception ont également été appréhendées (Dubois et Cornet, 2012 ; PoilpotRocaboy, Chevance et Dedessus-Le Moustier, 2014), ainsi que la souffrance psychique (Henrique Rogues de Souza et Iochins Grisci, 2005) et l'émotion au travail (Moulin et Mainhagu, 2013).

\section{Des perspectives salutogéniques marginales}

En revanche, les approches de type salutogénique restent limitées, les travaux s'orientant plus facilement vers des perspectives pathogéniques : ainsi, le bien-être au travail a principalement été abordé afin de clarifier le contenu même de ce concept (Colin et Grasser, 2011 ; Bergon, 2012 ; Creusier, 2013 ; Creusier, 2014 ; Cherkaoui et Montargot, 2014), Rodolphe Colle ayant étudié l'influence des temps de travail personnalisés sur le bien-être des salariés (Colle, 2005). D'autres approches «positives » de la SST ont vu le jour : la question de la qualité de l'emploi (Ilama, Belghiti-Mahut et Briole, 2010), ou plus largement de la santé au travail et de ses liens avec la performance (Stimec et Michel, 2010).

\section{Des concepts connexes à la SST toujours plus mobilisés}

Comme nous l'avons vu dans notre première partie, de nombreux concepts connexes à la SST ont été mobilisés durant les congrès de 1990 à 2000, cette tendance s'étant confirmée depuis. Ainsi, l'engagement au travail, la confiance, la satisfaction, l'implication, le sens du travail, la reconnaissance et l'équilibre vie professionnelle - vie privée font état d'un corpus théorique de plus en plus dense.

L'engagement au travail a été appréhendé à différents niveaux : les facteurs d'engagement au travail (Lapalme et Doucet, 2004; Hollet, 2006), les liens entre engagement affectif et pratiques de GRH (Chênevert, Charest et Simard, 2005 ; Weidmann et Gonin, 2013 ; Sriput, 2014), l'engagement organisationnel des jeunes cadres (Guerrero et al., 2005), l'engagement dans le travail selon la perception des caractéristiques et du contexte de travail (Carbonnel, 2006 ; Marcq, 2008), les liens entre engagement et efficacité au travail (Taktak Kallel, 2006), l'engagement et le soutien organisationnel (Chênevert, Vandenberghe et Tremblay, 2011) et enfin le désengagement au travail (Clergeau, Geffroy-Maronnat et Pihel, 2008 ; Christin et Moulette, 2013).

Concernant la confiance au travail, son rôle a été appréhendé dans l'amélioration de la relation d'emploi (Campoy et Neveu, 2005 ; Guerrero et Herrbach, 2007), dans la justice procédurale et le comportement organisationnel citoyen (Dolan, Tzafrir et Baruch, 2005), dans la médiation entre les pratiques RH et la performance prescrite (Tremblay, Cloutier et Chênevert, 2005), ou entre le leadership et l'engagement (Doucet, Simard et Tremblay, 2006), ainsi que son rôle dans le développement organisationnel (Amara et Bietry, 2008).

La satisfaction au travail a été étudiée au prisme de l'intéressement (Le Roux, 2000), de ses liens vis-à-vis de l'implication organisationnelle et de l'engagement des salariés à temps partiel ayant l'intention de départ (Palmero, 2000), la satisfaction des acteurs associatifs dans 
un contexte de changement (Chanut-Guieu, 2005), les liens entre satisfaction, engagement et hétérosexisme (Falcoz et Becuwe, 2009), la satisfaction et succès en carrière (Tremblay, Dahan et Gianecchini, 2009), celle des apprentis (Alves, Gosse et Sprimont, 2010), les relations ambigües entre satisfaction, implication et engagement (Besseyre des Horts et Nguyen, 2010), la satisfaction et l'implication des enseignants chercheurs (Drucker-Godard, Fouque, Gollety et Le Flanchec, 2012), et enfin les liens entre satisfaction et genre (Bencherqui, Kefi, Fronteau et Janand, 2014).

$\mathrm{Au}$ niveau de l'implication au travail, nous pouvons recenser l'analyse du concept d'implication (Biétry, 2012 ; Charles-Pauvers et Peyrat-Guillard, 2012), l'étude des relations entre implication et temps partiel (Palmero, 2001; Bonnet-Polese, 2003), implication et management à distance des cadres (Thévenet, 2001), l'implication des salariés comme clé de réussite du changement (Brasseur et Mzabi Héla, 2003), les relations entre implication et émotions au travail (Herrbach et Lérat-Pytlak, 2004), l'implication des salariés ayant vécu une période de restructuration (Naulleau, 2005 ; Duyck et Lahmouz, 2006 ; Lahmouz et Duyck, 2008), les liens entre implication et enrichissement travail/famille (Dumas, 2006), l'implication des professionnels de technologies d'information (Gutiérrez-Martinez, 2006 ; Gutiérrez-Martinez, 2009), l'implication des salariés en contexte d'interculturalité (Nkakleu, 2006 ; Nkakleu et Manga, 2010), la question des tensions de rôles et de l'implication (Commeiras, Loubès et Fournier, 2007), implication organisationnelle et pratiques de GRH (Gutiérrez-Martinez, 2007), implication et animal de compagnie (Chaudat, 2011), implication et responsabilité sociale (Closon et Leys, 2011), implication et effets de la pression budgétaire (Georgescu et Commeiras, 2011), implication et climat éthique (Golli, Swalhi et Yahiaoui, 2011), implication et souffrance (Safy, 2011), implication et syndicalisation (Elouaryarhli, 2014) et enfin implication et satisfaction des enseignants (Fournier, 2014). Nous pouvons également relever les travaux de Gregor Bouville sur le manque d'implication et l'absentéisme (Bouville, 2006).

Le sens du travail, ou sensemaking, a émergé dans les travaux de l'AGRH dès 2005 (Piccinini, Rocha de Oliveira, Dos Santos Fontoura et Schweig, 2005 ; Rocha de Oliveira et Piccinini, 2005) puis (Calvez, 2006; Dreyfuss, 2013), concept ensuite étudié en lien avec l'apprentissage organisationnel (Garreau, 2006), le changement organisationnel (Le RenduLizee, 2006; Guilmot et Vas, 2011) ou l'épuisement professionnel (Carrière et Bremner, 2011). Le manager en quête de sens a également été investigué (Rondeaux et Pichault, 2011), ainsi que le rôle des émotions dans le sensemaking (Garreau et Perrot, 2011) ou ses liens avec la RSE (El Bahri-Attou, Briole et Pijoan, 2014). La question de l'identité professionnelle a également été étudiée, dans les métiers de chercheurs (Leclercq et Potocki Malicet, 2006) ou dans ceux du soin (Georgescu et Rivière, 2013). Concernant la reconnaissance au travail, audelà des études sur la portée de ce concept (Bouzidi et El Akremi, 2006 ; Fall, 2011 ; Alves, Ardouin et Philippe, 2012), ce dernier a été analysé en fonction de ses liens avec l'isolement au travail (Belghiti-Mahut et Ortiz-Diaz, 2014) ou la reconnaissance du métier (JeannerodDumouchel, 2014)

Enfin, la question de l'équilibre travail - famille, ou de l'équilibre vie professionnelle - vie personnelle, a été appréhendée dans la continuité des travaux québécois présentés en première partie : nous pouvons ainsi citer l'étude exploratoire des pratiques d'équilibre travail - famille dans les entreprises françaises (Alis, Hourquet et Savigny, 1996) ou entre la France et la Grande-Bretagne (Sanséau et Smith, 2008), leur harmonisation (Alis et Dumas, 2003 ; Kilic, 2010), leur conflit négativement relié à la satisfaction au travail (Belghiti-Mahut, 2003 ; Belghiti-Mahut, 2013), leur conciliation inégalitaire (Alis et Dumas, 2005), les mesures 
d'aide à la conciliation travail - hors-travail (Barel et Frémeaux, 2005 ; Barel et Frémeaux, 2006), leur intégration professionnelle (Normand et Tremblay, 2005), la gestion du hors travail par les entreprises américaines (Ollier-Malaterre, 2005), la gestion du travail en contexte de conciliation travail - famille (Chrétien et Létourneau, 2006), l'articulation vie professionnelle - vie privée des couples à double carrière (Colle, Frimousse et Peretti, 2006 ; Colle et Christin, 2008), celle des cadres féminins (Deme, 2010), les variables déterminantes dans cette articulation (Tremblay, Najem et Paquet, 2006 ; Tremblay, 2010 ; Ballesteros Levya, Poilpot-Rocaboy et St-Onge, 2011), la perception des enjeux en matière d'équilibre vie professionnelle - vie personnelle selon les étapes de carrière (Naschberger, Quental et Legrand, 2013), les liens entre cet équilibre et l'implication organisationnelle (Garner, 2013).

\subsection{Les pratiques et acteurs en SST}

Au-delà des concepts de SST, la communauté AGRH a progressivement abordé les pratiques et les acteurs de la scène Santé et Sécurité au Travail tout en soulevant les problèmes de mesure associés à une meilleure compréhension de ces problématiques (Abord de Chatillon, 2004).

Ainsi, la gestion du stress a été abordée dès 2003 avec le cas des enseignants au sein d'une université tunisienne (Hechiche-Salah, Bousnina-Bouallegue et Touzani, 2003), gestion adaptée à la spécificité des sources de stress et conduisant à une réflexion autour des dispositifs RH à mettre en œuvre. La gestion des risques psychosociaux au travail, englobant diagnostic, prévention et résolution, a été plusieurs fois abordée (Douillet et Sahler, 2006 ; Chakor, 2011; Conjard, 2011; Gilbert, 2011; Attias-Delattre et Szpirglas, 2012 ; Durand, Krohmer et Baret, 2014), tandis que la prévention de la violence (Chapon, 2006), des troubles musculo-squelettiques (Bonnet, Savall et Zardet, 2006), du travail émotionnel (Alis, 2010) et le cas du coaching en SST (Nouamani et Briole, 2011) ont été abordés plus marginalement. D'autres pratiques ont été investiguées: les stratégies d'ajustement et de coping dans des situations de stress généré lors de fusions-acquisitions (Steiler et Rüling, 2003), l'usage du principe "pollueur payeur» dans le champ de la SST (Abord de Chatillon et Bachelard, 2005), les pratiques de RSE face au stress professionnel (Moisson et Roques, 2005) ainsi que le contrôle de gestion dans le domaine de la santé au travail (Bonnet et al., 2006).

Les pratiques en SST ont également été abordées dans une perspective plus proactive, sous la forme de guides et de préconisations formelles : l'idée de « savoir parler du travail » (Gambin et Lapeyrière, 2006), la proposition d'un processus d'intervention pour lutter contre le harcèlement psychologique au travail (Poilpot-Rocaboy et Winter, 2006), la co-production de nouvelles pratiques en management de la SST et l'usage de ces différents types de méthodologie (Carpentier et Bachelard, 2009), le management des hommes et de leur comportement en amont (Payre, 2010), l'évaluation de la bonne distance de l'individu à son organisation (Abord de Chatillon et Carrier Vernhet, 2012), l'apport de la méditation et de la pleine conscience (Françoise, 2012), l'intégration de l'éthique dans la co-construction des pratiques de GRH (Bachelard et Raïes, 2012), la prise en compte du genre dans l'intervention sur les conditions de travail (Chappert, 2013) et l'approche dialogique pour sensibiliser aux enjeux de SST (Bachelard, Beeler et Magakian, 2014).

Au niveau des acteurs de la SST, les travaux ont principalement gravité autour du manager, notamment le manager de proximité : l'analyse de la place du manager de proximité face au management de la SST (Carpentier, 2010; Clergeau et Pihel, 2010; Benion, 2013), les formations des managers pour les positionner comme acteurs de la santé mentale (Pezé et 
Journoud, 2011), leur soutien face aux tensions de rôle (Codo, 2012a), le stress des managers de proximité engagés dans leur travail (Codo, 2012b) attestent de cette place prépondérante. D'autres types d'acteurs ont été étudié : les consultants externes dans la prévention des RPS (Chakor, 2011) et les médecins du travail dans les actions de prévention primaire (Barel et Frémeaux, 2012).

\subsection{Les sources, facteurs et approches disciplinaires en SST}

Au-delà des concepts, pratiques et acteurs en SST, les travaux de chercheurs de l'AGRH se sont également articulés autour des sources, facteurs et approches disciplinaires de cette thématique.

Les principales sources sont d'ordre organisationnel: ainsi, de nombreux déterminants organisationnels de la santé au travail sont mis à jour, tels que l'utilisation des TIC (PeyratGuillard et Samier, 2001), l'insécurité dans l'emploi et ses conséquences sur les attitudes au travail et le bien-être des salariés (Leforestier-Schmidt, 2002), les transitions organisationnelles et les souffrances qu'elles provoquent (Dubouloy, 2002), les tensions de rôle et la surcharge de travail (Commeiras, Fournier et Loubès, 2003), les nouvelles formes d'organisation du travail (Cintas, 2003), le statut d'emploi et les comportements au travail (Saba et Lemire, 2004), la mise en danger par le travail en projet (Asquin, Garel et Picq, 2006), les déterminants managériaux de la santé au travail (Clergeau, Detchessahar, Devigne, Dumond, Honoré et Journé, 2006 ; Clergeau, Geffroy-Maronnat et Pihel, 2007 ; Stimec, Bertrand, Michel et Detchessahar, 2007), ses déterminants socio-psychologiques (Garcias, Dalmasso et Sardas, 2011), les modes de régulation du travail et leurs impacts sur la santé des salariés (Detchessahar, Devigne et Stimec, 2008) le lien entre pratiques managériales et santé au travail (Honoré, 2008) et le «tournant gestionnaire» des établissements de santé (Detchessahar et Grevin, 2009).

D’autres sources sont abordées de manière plus spécifiques et pathogéniques : les facteurs de stress d'ordre organisationnel (Benedetto-Meyer et Illyine, 2006), les modèles explicatifs des causes de l'absentéisme (Dumond, 2006 ; Bouville, 2007 ; Ndao et Diop, 2008 ; Bouville, 2010), le déséquilibre effort/récompense comme source de stress et d'intention de départ (Colle et Moisson, 2007), les déterminants du stress et de l'épuisement professionnel (Moisson, Fuhrer et Cucchi, 2010), ceux du harcèlement moral (Bouville et Campoy, 2011), de la souffrance psychosociale (Abord de Chatillon, Carrier Vernhet et Desmarais, 2010), le caractère pathogène du nouveau management public (Abord de Chatillon et Desmarais, 2011), la pression à l'activité comme source de stress et d'épuisement professionnel (Commeiras et Georgescu, 2012), les rythmes atypiques, (Coulon, Schoenenberger, Gilibert, Banovic et El Haddad, 2013), les facteurs de stress en secteur médico-social (Vinot, 2013), les effets de la lean production sur la santé au travail (Bouville et Schmidt, 2014). L'approche salutogénique des sources et facteurs reste, à l'instar des concepts salutogéniques, marginale : on peut ainsi recenser les études portant sur les aspects organisationnels et le bien-être au travail (Colin et Grasser, 2011), sur les conditions organisationnelles et managériales favorisant l'émergence d'un environnement favorable à la construction de la santé (Benion, 2010) et sur le sport en entreprise comme nouvel espace de santé au travail (Chakor, Calvin, Dimeglio et Dantin, 2014). Enfin, certaines recherches se sont intéressées aux perceptions des individus dans le management de la SST : les croyances dans le management de la sécurité et les perceptions de risque (Kouabenan, 2008), la compréhension de la SST dans les entreprises chinoises (Duport et Janicot, 2011), et la remise en question de l'effet Hawthorne (Geoffroy, 2013). 
Le champ de recherche de la SST au sein de l'AGRH a également vu croître certaines approches pluridisciplinaires : l'apport de l'ergonomie et de la psychologie du travail dans l'analyse du travail, de son organisation et de son contenu (Rogard et Vallery, 1991 ; Gambin et Lapeyriere, 2006 ; Suquet, 2006), l'approche psychologique de la saturation des salariés (Johnson, Giraud, Autissier et Bareil, 2014), la clinique du travail et l'accompagnement des salariés en souffrance, avec des réflexions autour du coaching d'inspiration psychanalytique (Arnaud et Guinchard, 2005), l'utilisation de la psychanalyse en GRH (Arnaud, 2005 ; Naulleau, 2005; Arnaud et Guinchard, 2006), l'approche épidémiologique de la reconnaissance au travail (Boitel, Castel, Delgoulet, Jabot, Juillard et Presseq, 2006) et l'approche économique des troubles musculo-squelettiques (Douillet, Fauconnier, Pepin et Schweitzer, 2006). Parallèlement à ces approches disciplinaires, il est intéressant de montrer comment ces dernières ont pu faire évoluer les manières d'appréhender et de conceptualiser l'entreprise : ainsi, l'organisation a pu être analysée comme une somme de nœuds de contrats psychologiques (De Bry et Galindo, 2005; Castaing et Roussel, 2006 ; Desmarais et Dubouloy, 2010; Lamargot et Peyrat-Guillard, 2014) et les individus comme pouvant s'appuyer sur des ressources spécifiques, notamment psychologiques (Edey-Gamassou, 2005).

\subsection{La mesure en SST}

L'idée de mesure s'est, logiquement, développée à partir du congrès de Montréal datant de 2004 et intitulé «La GRH mesurée! »: depuis, de nombreuses propositions d'échelles de mesure ont émergé. Ainsi, nous avons relevé : la mesure de la dissonance émotionnelle au travail, de son contenu et des effets engendrés (Van Hoorebeke, 2004), la proposition d'un instrument de mesure du contrat psychologique (Guerrero, 2004), l'impact de la perception de rupture du contrat psychologique (Lapalme, Simard et Tremblay, 2006), la proposition d'une échelle de mesure multidimensionnelle de la confiance du salarié vis-à-vis de son entreprise (Benraïss et Meyssonnier, 2005), la mesure de l'engagement affectif et normatif (Foucher, Morin, Bentein et Trottier, 2004 ; Quenneville, Simard et Rabouin, 2004), la validation d'une échelle d'engagement au travail (Hollet, 2005), la mesure de l'implication organisationnelle et la perception du soutien organisationnel (Dumas, Alis et Campoy, 2004), une réflexion en faveur d'une nouvelle échelle de mesure de l'implication dans le métier (Demery-Lebrun, 2006), la mesure de l'implication organisationnelle (Demery-Lebrun, 2007 ; Cognat et Sardas, 2014). Concernant la satisfaction au travail, différentes échelles de mesure et modes de calcul ont également été proposées : la mesure du concept de satisfaction au travail (Mignonac, 2004), celle des effets modérateurs de la satisfaction, ou de la souffrance au travail, en fonction du temps de travail (Abord de Chatillon \& Desmarais, 2007), le test d'un modèle autour de la satisfaction des salariés (Alves, Gosse et Sprimont, 2009), la mesure combinée du soutien, de la confiance, de la satisfaction et de l'engagement organisationnel affectif (Galois, 2007), la mesure de l'effet médiateur du niveau de mobilisation dans la relation satisfaction/absentéisme et de l'effet modérateur de la dispersion (Tremblay, Lero et Larivière, 2013). Au niveau du soutien social : la construction d'une échelle de la perception du soutien social (Ruiller, 2007), le développement d'une échelle de mesure du soutien social (Ruiller, 2008), la mesure de l'impact du support organisationnel perçu sur le stress (Wuidar, Stinglhamber et Hanin, 2009). D'autres éléments, divers et variés, ont été l'objet de mesures : la mesure de l'impact des stresseurs organisationnels sur le bien-être dans un contexte de flexibilité (El Akremi, Haddaji et Sassi, 2006), de l'impact des tensions de rôle (Commeiras, Loubès et Fournier, 2007 ; Codo, 2011), la mesure de la fidélité organisationnelle (Peretti et Swalhi, 2007), de l'exposition au harcèlement psychologique (Poilpot-Rocaboy, Notelaers et 
Hauge, 2009), du sentiment de reconnaissance au travail (Terramorsi et Peretti, 2010), du rôle du climat général de formation sur l'engagement affectif organisationnel et le BE des salariés (Bergon, 2012), celle du développement personnel (Jaotombo et Brasseur, 2013), des effets des conditions de travail sur la qualité de service perçue (Mansour et Commeiras, 2014) et des déterminants de l'absentéisme en milieu hospitalier (Banville et Chênevert, 2006). Le modèle de Karasek a également été "revisité » par les régressions polynomiales (Guénette, Lee et Papart, 2006). Enfin, certaines communications ont placé au cœur de leurs études les analyses des modalités et échelles de mesure : celles du stress (Hollet-Haudebert, Edey-Gamassou et Allard-Poesi, 2010 ; Allard-Poesi, Edey-Gamassou et Hollet-Haudebert, 2011) et de la satisfaction (Iglesias, Renaud et Tschan, 2010).

\section{Conclusion : Les perspectives futures de recherche en SST au sein de la communauté AGRH - Vers l'ouverture d'une phase d'approfondissement?}

Au-delà des limites d'un tel exercice, l'observation des travaux réalisés ces vingt-cinq dernières années décrit à la fois la difficulté visant à limiter un champ scientifique, mais également des tendances de fond qui offrent des perspectives de recherche intéressantes.

Ce travail comporte un ensemble de limites qu'il convient de préciser. D'une part, les auteurs ont effectué des choix quant aux contours du champ de la SST, et d'autre part ils ont fondé leur travail sur deux à trois lectures de chacun des textes concernés, sans pour autant faire appel à un logiciel d'analyse de contenu. Si ce choix est discutable, il nous a permis de saisir en priorité le sens général du contenu des communications en SST, sans forcément présenter les spécificités des contenus de chacune d'entre elles. En effet, le propos n'était pas de rendre compte de l'intégralité de ce qui a été dit, mais plutôt de tenter de saisir les logiques globales et leurs enjeux.

Notre analyse a ainsi permis de mettre en relief un champ qui possède des contours variables, mais également des chercheurs aux positionnements évolutifs dans leurs choix paradigmatiques. Le thème de la Santé et Sécurité au Travail, absent puis sous-entendu, est aujourd'hui au cœur des problématiques théoriques débattues au sein de l'AGRH. Cette extension «numérique »s'accompagne d'une extension conceptuelle : ce sont désormais les thématiques traditionnelles de la GRH qui incorporent des composantes SST ou font le choix d'examiner les liens pouvant exister avec les indicateurs de la santé au travail. Si cela est aussi important aujourd'hui, c'est également parce que les problèmes de SST sont à l'agenda des organisations et qu'elles sont prêtes à accueillir sur ce thème des chercheurs pour mieux comprendre et améliorer le fonctionnement de leurs organisations. À la sempiternelle question « comment mesurer la performance de l'action et des outils RH? ", la thématique de la SST fournit aussi bien un cadre conceptuel que des outils de mesure permettant de rendre compte de l'état de la population au travail. On observe ainsi un glissement des préoccupations des chercheurs en GRH qui accompagnent le mouvement de la fonction $\mathrm{RH}$, s'éloignant des processus stricts de gestion de la fonction (formation, rémunération, évaluation, motivation etc.) vers un approfondissement de la question du travail et de l'activité. En cela, les objets d'analyse et d'observation amènent progressivement les chercheurs en GRH vers leurs collègues des autres disciplines des sciences du travail (ergonomie, psychologie du travail, sociologie du travail, etc.). Cette analyse dresse également un panorama des thématiques de la SST. Ainsi, si les questions de sécurité ont été les premières à être prises en compte à la fois par les auteurs européens et nord-américains, la thématique des pathologies psychosociales s'est imposée en quelques années. À la question quasiment unique du stress se sont ajoutées 
les RPS, les violences (et notamment le harcèlement), puis l'épuisement professionnel et enfin la question du bien-être.

Mais au-delà de ces constats, il convient de poser, au vu du passé, ce que pourrait être l'avenir de ce champ de recherche. Trois axes paraissent pouvoir être dessinés :

- un premier axe autour de l'analyse des pratiques et outils de gestion de la SST cherchant à définir ce qui peut être fait et ce qui fonctionne. Nous retrouvons notamment ici la problématique de l'audit social, en particulier des pratiques de prévention de la SST. Il s'agit d'intégrer les dimensions organisationnelles, managériales et technologiques au sein de la stratégie d'entreprise. Par-delà les démarches et outils comme le document unique (conçu sur une logique technico médicale), comment appréhender les conséquences internes (corrélation entre santé des salariés et santé économique de l'entreprise) et externes (en particulier en terme financier, social et humain) de celles-ci ?;

- un deuxième axe autour de l'articulation de la SST avec les autres pratiques de GRH nous incite à poser la question des missions de la fonction RH. Les différents travaux cités nous montrent qu'il est contreproductif pour un DRH de construire en parallèle des dispositifs plus ou moins complémentaires pour traiter les différentes problématiques auxquelles l'entreprise est confrontée (santé au travail, gestion des âges, gestion de l'égalité professionnelle...). Repenser la GRH pour tenir compte des évolutions réglementaires (allongement de la durée de vie au travail, cumul emploi retraite, contrat de génération, prévention de la pénibilité, etc.), évolutions démographiques (natalité forte, espérance de vie en hausse, vieillissement de la population, etc.) et évolutions sociologiques (ouverture sur le monde, regard sur le travail, diversité et faits religieux, etc.). Les enjeux sont nombreux et d'importance : financiers (coûts directs et indirects, productivité, etc.), sociaux (problématique de l'inaptitude et de l'insuffisance professionnelle, donc de l'employabilité durable), humain (si le nombre de décès par accident du travail est stable, celui des maladies professionnelles reconnues explose, alors même que le législateur s'empare de questions comme celles du burn out). La GRH ne peut plus être la même et les chercheurs en GRH ont un rôle déterminant à jouer, à condition de produire des connaissances actionnables ;

- et enfin, un troisième axe autour de l'approfondissement des modèles théoriques d'appréhension des questions de SST. Pour cela, les chercheurs doivent intégrer $a$ minima quatre niveaux de logique : une logique de finalité (le projet commun des différentes parties prenantes), une logique d'organisation (comment), une logique d'arbitrage (avec les acteurs, ce qui se gagne d'un côté, ne se perd pas forcément de l'autre), et une logique de discussion (centrée sur le travail avec des espaces prévus à cet effet).

Ainsi, vingt-cinq ans après la création de l'AGRH, la thématique SST accompagne les évolutions de la fonction et participe au renouvellement non seulement des représentations sociales de la fonction, mais aussi des cadres d'analyse des situations de travail. Si la fonction RH est toujours à la recherche de la performance organisationnelle, les questions de SST traitées par les chercheurs de l'association ont participé à leur manière à une évolution de la fonction et de la recherche vers une meilleure connaissance de l'activité des acteurs de nos 
organisations. Aujourd'hui, au-delà des trois axes énoncés, de multiples pistes de travail s'offrent aux chercheurs en SST : comment repenser les méthodes de mise en place de nouvelles normes dans un contexte de bureaucratisation des démarches SST ? Comment peuton «faire mieux» sans pour autant alourdir la structuration réglementaire de nos organisations ? Au niveau du concept même de travail et d'activité de travail, comment le « revisiter» au profit de la GRH et des acteurs de la scène SST ? Quels sont les apports des trois aspects complémentaires du travail (subjectif, objectif, collectif) à nos questionnements, notamment en termes de relation au travail, de construction personnelle, de gestion de carrière, de cycle de vie professionnelle, etc. ? (Autier et Ramboatiana, 2014). Nous rejoignions ainsi le travail de François Hubault (2006) : "le défi est de dépasser l'ambiguïté gestionnaire du terme activité et d'assumer les conséquences de la reconnaissance de la puissance productive de la subjectivité ».

\section{Auteurs ayant directement contribué au champ de la SST à partir de leur(s) communication(s) au sein des congrès de l'AGRH}

\begin{tabular}{|c|c|}
\hline Auteur & $\begin{array}{l}\text { Année(s) de } \\
\text { contribution } \\
\text { SST }\end{array}$ \\
\hline Abbas S.G. & 13 \\
\hline $\begin{array}{l}\text { Abord de } \\
\text { Chatillon E. }\end{array}$ & $\begin{array}{l}92,96,97, \\
98,99,04, \\
05,07,09, \\
10,11,12\end{array}$ \\
\hline Alis D. & $96,03,05,10$ \\
\hline $\begin{array}{l}\text { Allard-Poesi } \\
\text { F. }\end{array}$ & 10,11 \\
\hline Arnaud G. & 05 \\
\hline Asquin A. & 06 \\
\hline $\begin{array}{l}\text { Attias- } \\
\text { Delattre V. }\end{array}$ & 12 \\
\hline Aubert N. & 91 \\
\hline Bachelard O. & $\begin{array}{l}04,05,09, \\
12,14\end{array}$ \\
\hline $\begin{array}{l}\text { Ballesteros } \\
\text { L. }\end{array}$ & 11 \\
\hline Banovic I. & 13 \\
\hline Banville B & 06 \\
\hline Barel Y. & $05,06,12$ \\
\hline Baret C. & 14 \\
\hline Bartoli M. & 90 \\
\hline Beaucourt C. & 90 \\
\hline
\end{tabular}

\begin{tabular}{|c|c|}
\hline Beeler B. & 14 \\
\hline $\begin{array}{l}\text { Belghiti- } \\
\text { Mahut S. }\end{array}$ & $03,13,14$ \\
\hline Benion A. & 10,13 \\
\hline Bergon G. & 12 \\
\hline $\begin{array}{l}\text { Bernatchez } \\
\text { J.-C. }\end{array}$ & 91 \\
\hline Bertrand $\mathrm{T}$. & 07 \\
\hline $\begin{array}{l}\text { Bennedett- } \\
\text { Meyer M. }\end{array}$ & 06 \\
\hline Bonnet M. & 06 \\
\hline $\begin{array}{l}\text { Bonnet- } \\
\text { Polese I. }\end{array}$ & 03 \\
\hline $\begin{array}{l}\text { Bousnina- } \\
\text { Bouallegue } \\
\text { Z. }\end{array}$ & 03 \\
\hline Bouteiller D. & 94 \\
\hline Bouville $\mathrm{G}$ & $\begin{array}{l}06,07,10, \\
11,14\end{array}$ \\
\hline Bremner N. & 11 \\
\hline Briole A. & 11 \\
\hline Cadieux J. & 04 \\
\hline Calvin S. & 14 \\
\hline Campoy E. & 11 \\
\hline Carpentier S. & $04,09,10$ \\
\hline
\end{tabular}

\begin{tabular}{|l|l|}
\hline $\begin{array}{l}\text { Carrier } \\
\text { Vernhet A. }\end{array}$ & $10,12,12$ \\
\hline Carrière J. & 11 \\
\hline Chakor T. & $11,14,14$ \\
\hline Champion I & 06 \\
\hline Chapon E. & 06 \\
\hline Chappert F. & 13 \\
\hline Chaudat P. & 11 \\
\hline Chênevert D. & 06,11 \\
\hline $\begin{array}{l}\text { Cherkaoui } \\
\text { W. }\end{array}$ & 13,14 \\
\hline Chevance A. & 14 \\
\hline Chretien L. & 06 \\
\hline Christin J. & 08,13 \\
\hline Cintas C. & $03,06,11$ \\
\hline Clergeau C. & $06,07,08,10$ \\
\hline Codo S. & $10,11,12,12$ \\
\hline Cognat A.S. & 14 \\
\hline Colin T. & $11,14,12$, \\
\hline Commeiras P. & $03,07,08$ \\
\hline C. & 11 \\
\hline C. & \\
\hline C. & \\
\hline C. & \\
\hline
\end{tabular}




\begin{tabular}{|c|c|}
\hline Cornet A. & 12 \\
\hline Coulon R. & 13 \\
\hline $\begin{array}{l}\text { Cousineau } \\
\text { M. }\end{array}$ & 14 \\
\hline Creusier J. & 13,14 \\
\hline Cucchi A. & 10 \\
\hline Dalmasso C. & 11 \\
\hline Dantin P. & 14 \\
\hline $\begin{array}{l}\text { Dedessus-Le- } \\
\text { Moustier N. }\end{array}$ & 14 \\
\hline Deharo G. & 13 \\
\hline Desmarais C & $\begin{array}{l}07,10,10, \\
11,12\end{array}$ \\
\hline Desmarais L. & 04 \\
\hline $\begin{array}{l}\text { Detchessahar } \\
\text { M. }\end{array}$ & $06,07,08,09$ \\
\hline Devigne M. & 06,08 \\
\hline Dimeglio I. & 14 \\
\hline $\begin{array}{l}\text { Dionne- } \\
\text { Proulx J. }\end{array}$ & 91 \\
\hline Diop B. & 08 \\
\hline Douillet P. & 06,06 \\
\hline Dubois V. & 12 \\
\hline Dubouloy M. & 02,10 \\
\hline Dumas M. & $03,05,06$ \\
\hline Dumond J.P. & 06,06 \\
\hline Duport M. & 11 \\
\hline Durand S. & 14 \\
\hline $\begin{array}{l}\text { Edey- } \\
\text { Gamassou C. }\end{array}$ & $\begin{array}{l}03,05,08, \\
10,11\end{array}$ \\
\hline El Akremi A. & 05,06 \\
\hline El Haddad N. & 13 \\
\hline $\begin{array}{l}\text { Fauconnier } \\
\text { D. }\end{array}$ & 06 \\
\hline Fournier C. & 03,07 \\
\hline Françoise D. & 12 \\
\hline Fremeaux S. & $05,06,12$ \\
\hline Frimousse $\mathrm{S}$. & 06,13 \\
\hline Fuhrer C. & 10 \\
\hline
\end{tabular}

\begin{tabular}{|c|c|}
\hline Garcias F. & 11 \\
\hline Garel G. & 06 \\
\hline Garner P. & 13 \\
\hline $\begin{array}{l}\text { Geffroy- } \\
\text { Maronnat B. }\end{array}$ & 07,08 \\
\hline Gilbert P. & 11 \\
\hline Gilibert D. & 13 \\
\hline Giorgescu I. & 12 \\
\hline Grasser B. & 11 \\
\hline Grevin A. & 09 \\
\hline $\begin{array}{l}\text { Guenette } \\
\text { A.M. }\end{array}$ & 06 \\
\hline Guérin G. & $93,93,94$ \\
\hline Guerrero S & 07 \\
\hline Guinchard R. & 05 \\
\hline Haddaji N. & 06 \\
\hline Haines V. & 94 \\
\hline Hanin D. & 09 \\
\hline Hauge L.J. & 09 \\
\hline $\begin{array}{l}\text { Hechiche- } \\
\text { Salah L }\end{array}$ & 03 \\
\hline Heerbach O. & 07 \\
\hline $\begin{array}{l}\text { Henrique } \\
\text { Rogues de } \\
\text { Souza P. }\end{array}$ & 05 \\
\hline $\begin{array}{l}\text { Hofaidhllaou } \\
\text { i M. }\end{array}$ & 13 \\
\hline $\begin{array}{l}\text { Hollet } \\
\text { Audebert S. }\end{array}$ & $05,10,11$ \\
\hline Honoré L. & 06,08 \\
\hline $\begin{array}{l}\text { Hourquet P. } \\
\text { G. }\end{array}$ & 96 \\
\hline Illyne L. & 06 \\
\hline $\begin{array}{l}\text { Iochins } \\
\text { Grisci C. }\end{array}$ & 05 \\
\hline Janicot L. & 11 \\
\hline Journé B. & 06 \\
\hline Journoud S. & 11 \\
\hline Kilic S. & 10 \\
\hline Kouabenan & 08 \\
\hline
\end{tabular}

\begin{tabular}{|c|c|}
\hline D.R. & \\
\hline Krohmer C. & 14 \\
\hline Langlois V. & 14 \\
\hline Larivière M. & 13 \\
\hline Lee Y.T. & 06 \\
\hline $\begin{array}{l}\text { Leforestier- } \\
\text { Scmidt I }\end{array}$ & 02 \\
\hline Legrand C. & 13 \\
\hline Lemire L. & 04 \\
\hline Lero D. & 13 \\
\hline Létourneau I. & 06 \\
\hline Loubès A. & 03,07 \\
\hline $\begin{array}{l}\text { Magakian } \\
\text { J.L. }\end{array}$ & 14 \\
\hline Mansour S. & $12,13,14$ \\
\hline Michel X. & 07,10 \\
\hline Moisson V. & $05,07,08,10$ \\
\hline Molines M. & 10 \\
\hline Montargot N. & 13,14 \\
\hline Moulette P. & 13 \\
\hline Najem E. & 06 \\
\hline $\begin{array}{l}\text { Naschberger } \\
\text { C. }\end{array}$ & 13 \\
\hline Ndao A. & 08 \\
\hline Neveu J.P. & $96,06,09$ \\
\hline Normand P. & 05 \\
\hline Notelaers G. & 09 \\
\hline Nouamani S. & 11 \\
\hline $\begin{array}{l}\text { Ollier- } \\
\text { Malaterre A. }\end{array}$ & 05 \\
\hline Ortiz-Diaz S. & 14 \\
\hline Paquet R. & 06 \\
\hline Papart J.P. & 06 \\
\hline Paturel D. & 04,06 \\
\hline Payre S. & 10 \\
\hline Pepin M. & 06 \\
\hline Peretti J.M. & 06,13 \\
\hline
\end{tabular}




\begin{tabular}{|l|l|}
\hline Pezé S. & 10,11 \\
\hline Picard D. & 00 \\
\hline Picq T. & 06 \\
\hline Pihel L. & $07,08,10$ \\
\hline $\begin{array}{l}\text { Poilpot- } \\
\text { Rocaboy G. }\end{array}$ & $\begin{array}{l}94,98,02, \\
06,09,11,14\end{array}$ \\
\hline Point S. & 13 \\
\hline Poirot M. & 04,05 \\
\hline Quental C. & 13 \\
\hline Raies K. & 12 \\
\hline Rogard V. & 91 \\
\hline Roger A. & $94,95,13$ \\
\hline Roques O. & $94,95,96$, \\
& $00,01,05,09$ \\
\hline Roy M. & 04 \\
\hline Ruiller C. & 07,08 \\
\hline Rüling C.C. & 03 \\
\hline Saba T. & 04 \\
\hline Safy F. & 11 \\
\hline Sanséau P.Y. & 08 \\
\hline
\end{tabular}

\begin{tabular}{|l|l|}
\hline St Onge S. & 93,11 \\
\hline Sahler B. & 06 \\
\hline Sardas J.C. & 11,14 \\
\hline Sassi N. & 05,06 \\
\hline Savall H. & 06 \\
\hline Savigny X. & 96 \\
\hline Schmidt C. & 14 \\
\hline $\begin{array}{l}\text { Schoenenber } \\
\text { ger S. }\end{array}$ & 13 \\
\hline $\begin{array}{l}\text { Schweitzer } \\
\text { J.M. }\end{array}$ & 06 \\
\hline $\begin{array}{l}\text { Serrano } \\
\text { Archimi C. }\end{array}$ & 09 \\
\hline Simard M. & 93 \\
\hline Smith M. & 08 \\
\hline $\begin{array}{l}\text { Sprimont } \\
\text { P.A. }\end{array}$ & 11 \\
\hline Steiler D. & 03 \\
\hline Stimec A. & $07,08,10$ \\
\hline $\begin{array}{l}\text { Stinglhamber } \\
\text { F. }\end{array}$ & 09 \\
\hline Swailhi A. & 13 \\
\hline
\end{tabular}

\begin{tabular}{|l|l|}
\hline Szpirglas M. & 12 \\
\hline Touzani M. & 03 \\
\hline $\begin{array}{l}\text { Tremblay } \\
\text { D.G. }\end{array}$ & $05,06,10,13$ \\
\hline Tremblay M. & 11 \\
\hline Trottier R. & 93 \\
\hline Valleiry G. & 91 \\
\hline $\begin{array}{l}\text { Van } \\
\text { Hoorebeke } \\
\text { D. }\end{array}$ & 04 \\
\hline $\begin{array}{l}\text { Vandenbergh } \\
\text { e C. }\end{array}$ & 11 \\
\hline Vauclin S. & 99 \\
\hline Vinot A. & 13 \\
\hline Wills & 93 \\
\hline Winter R. & 06 \\
\hline Wuidar D. & 09 \\
\hline Zardet V. & 06 \\
\hline Zgoulli S. & 13 \\
\hline
\end{tabular}

\section{Bibliographie issue des Actes des Congrès de l'AGRH :}

\begin{tabular}{|l|}
\hline Actes du Congrès de l'AGRH, 1990, Bordeaux \\
\hline Amado G., Deumie C., (1990). « Perspectives de l'entreprise et recherche en G.R.H. », Séance plénière. \\
\hline $\begin{array}{l}\text { Bartoli M., « Gestion des ressources humaines ou du travail humain ? Questions d'ordre méthodologique et pratique sur la fonction RH } \\
\text { dans l'entreprise » }\end{array}$ \\
\hline Beaucourt C., « Pour une gestion sociale ajustée » \\
\hline Bosquet R., « Allocution d'ouverture du président de l'A.N.D.C.P. » \\
\hline Bournois F., « La place de la fonction Ressources Humaines en Europe : similitudes et différences » \\
\hline Brabet J., Fenneteau H., « La fonction Personnel : évolutions et différenciations » \\
\hline Delperier A., Rapport de la Commission « Développement de l'organisation et stratégies sociales » \\
\hline Duyck J.-Y., « Les métiers de la fonction Ressources Humaines. Appellations, missions et profils » \\
\hline Gosselin L., « La fonction Ressources Humaines : modèle et typologie » \\
\hline Igalens J., « Allocution d'ouverture du président de l'AGRH » \\
\hline Louart P., « Trois paradoxes en gestion des Ressources Humaines » \\
\hline Noel X., « Flux de personnels et achats de services » \\
\hline Pouget M., Rapport de la Commission « L'évolution des hommes de la fonction » \\
\hline $\begin{array}{l}\text { Roger A., « De la gestion du personnel à la gestion des ressources humaines. Une analyse de l'évolution de la fonction Personnel à travers } \\
\text { les offres d'emploi » }\end{array}$ \\
\hline
\end{tabular}


Sire B., Séance plénière, « Ouverture »

Vignon C., Rapport de la Commission « Autres regards sur la GRH »

Weiss D., Séance plénière, « Recherches sur la fonction et fonctions de la recherche en GRH »

\section{Actes du Congrès de l'AGRH, 1991, Cergy}

Aktouf O., " Mobilisation des personnes, management et culture d'entreprise »

Aubert N., « Le coût de l'excellence ou la face d'ombre des entreprises conquérantes »

Bernard A., « Implication, paramètres personnels et adhésion aux politiques d'entreprise »

Bernatchez J.-C., « La satisfaction à l'égard du régime de conditions de travail chez les cadres intermédiaires. Des milieux «à vision » dans le secteur québécois de la santé »

Dionne-Proulx J., « La promotion de la santé au travail, un outil de gestion »

Duyck J.-Y., « Le vocabulaire de la GRH. Analyse comparative du langage des chercheurs et des praticiens »

Igalens J. et Penan H., « La structure des connaissances en gestion des ressources humaines. Identification des programmes de recherche »

Neveu J.-P., « Méthodologie de l'implication »

Rogard V. et Vallery G., "GRH et analyse ergonomique du travail dans la banque et l'assurance : une approche des changements socioorganisationnels »

Tissier-Desbordes E. et Thevenet M., « Implication dans le produit et implication dans l'organisation »

\section{Actes du Congrès de l'AGRH, 1992, Lille}

Abord de Chatillon E., « L'accident du travail peut-il se résumer à une non-qualité ?»

\section{Actes du Congrès de l'AGRH, 1993, Jouy-en-Josas}

Fiol M. et Solé A., «L’inexprimable besoin des managers. Entreprise et sens »

Peyrat D., «Pratiques de management des ressources humaines et implication des salariés: l'importance de la contingence organisationnelle »

St-Onge S., Guérin G., Trottier R., Simard M. et Haines V., « L’équilibre travail-famille : un enjeu organisationnel »

Wils T. et Guérin G., « Les pratiques de ressources humaines les plus réductrices du malaise des ingénieurs syndiqués »

\section{Actes du Congrès de l'AGRH, 1994, Montpellier}

Bouteiller D., «Gestion participative et santé-sécurité du travail: une exploration de la dynamique du pouvoir au sein de la ligne hiérarchique »

Guérin G., St-Onge S., Wils T., Haines V., Trottier R., Simard M., « Profils des organisations ayant implanté au Québec des pratiques d'aide à la gestion de l'équilibre travail-famille (ETF) »

Poilpot-Rocaboy G., «Un modèle de la divergence de la satisfaction des individus à l'égard de la protection sociale complémentaire d'entreprise : un essai de validation des hypothèses théoriques formulées »

Roger A. et Roques O., " Variation du stress perçu aux différentes étapes de carrière des cadres »

Roussel P., « Méthode de validation de questionnaire en GRH : l'exemple du QSR en phase exploratoire - Questionnaire de Satisfaction à l'égard de la rémunération »

\section{Actes du Congrès de l'AGRH, 1995, Poitiers}

Berman J. A., « Caractéristiques de travail, problèmes de gestion, attributs du personnel et la satisfaction de travail des programmeurs »

Roger A. et Roques O., « Le stress au travail des cadres : déterminants et modes de réaction »

\section{Actes du Congrès de l'AGRH, 1996, Paris}

Abord de Chatillon E., «Une évaluation du lien entre performance et gestion de la sécurité du travail : la situation du décolletage dans le secteur de la vallée de l'Arve»

Alis D., Hourquet P.-G. et Savigny X., «Étude exploratoire des pratiques d'équilibre travail-famille dans les entreprises françaises » 
Neveu J.-P., « Performance et épuisement professionnel : ingénierie de la santé psychologique au travail »

Paillé P., « Implication, comportement de retrait et satisfaction des acteurs. Le cas d'un centre hospitalier d'une ville de province »

Roques O., «Les réactions des salariés aux transitions de carrière : une approche en termes de stress au travail »

\section{Actes du Congrès de l'AGRH, 1997, Montréal}

Abord de Chatillon E., «Comment améliorer la gestion des comportements et attitudes des acteurs face au problème de la sécurité du travail ? : la méthode RECORE »

Kopel S. et Sire B., « Mise en évidence des facteurs de satisfaction des salariés sous statut de la fonction publique. L'exemple d'une DDE »

\section{Actes du Congrès de l'AGRH, 1998, Versailles}

Abord de Chatillon E., « Management de la sécurité du travail : une comparaison entre l'usine de Montréal d'Imperial Tobacco et celle de Riom de la SEITA »

Legault G. et Simard G., « Satisfaction au travail et sentiment d'appartenance : une relation à explorer »

Neveu J.-P. et Peyrat-Guillard D., «L'implication des femmes-cadres : entre éclatement et cohérences »

Poilpot-Rocaboy G., «La fonction GRH comme garant du respect du « contrat psychologique relationnel » dans l'entreprise : le cas du harcèlement professionnel»

\section{Actes du Congrès de l'AGRH, 1999, Lyon}

Abord de Chatillon E. et Vauclin S., « La valse hésitation de l'autonomie et du contrôle en management de la sécurité au travail »

\section{Actes du Congrès de l'AGRH, 2000, Paris}

Le Roux A., « La satisfaction des salariés à l'égard de l'intéressement : une adaptation du modèle de Florkowski et Schuster au contexte français »

Palmero S., «Implication organisationnelle, satisfaction au travail, engagement au travail et intention de départ des salariés à temps partiel »

Picard D., «Analyse comparée des travaux anglo-saxons sur le stress professionnel et des recherches de l'école française de psychodynamique du travail : quelles conciliations envisager? »

Roques O., «L'influence du lieu de contrôle, du type A ou B et de l'étape de carrière sur le stress au travail : à la recherche d'une confirmation internationale $»$

\section{Actes du Congrès de l'AGRH, 2001, Liège}

Palmero S., « Les effets de types de travail à temps partiel sur l'implication organisationnelle »

Peyrat-Guillard D. et Samier N., « Utilisation des TIC et pratiques de GRH associées : impact perçu sur les attitudes et les comportements des salariés »

Roques O., « Stress et travail : quoi de neuf docteur ? Étude de huit cas d'entreprises suivis par des médecins du travail »

Thévenet M., «Implication dans l'entreprise et dimension relationnelle du travail : des enseignements pour le management à distance »

\section{Actes du Congrès de l'AGRH, 2002, Nantes}

Dubouloy M., « Gérer les transitions organisationnelles et les souffrances qu'elles provoquent. Exemple d'un dispositif « déconstruction et reconstruction »" »

Leforestier-Schmidt I., «L'insécurité dans l'emploi dans un contexte organisationnel positif. Conséquences sur les attitudes au travail et le bien-être des salariés »

Poilpot-Rocaboy G., « Harcèlement psychologique au travail : une " pratique de GRH » au service des stratégies de croissance externe »

\section{Actes du Congrès de l'AGRH, 2003, Grenoble}

Alis D. et Dumas M., « 35 heures, soutien organisationnel perçu et harmonisation vie familiale/vie professionnelle »

Belghiti-Mahut S., « La relation entre le conflit vie professionnelle/vie familiale et la satisfaction au travail : une investigation empirique »

Bonnet-Polese I., « Une recherche exploratoire du rapport au travail des cadres à temps partiel et de leur implication au travail : l'influence de l'environnement immédiat de travail» 
Brasseur M. et Mzabi Héla J., «L'implication des salariés, clef de réussite du changement? »

Cintas C., « Nouvelles formes d'organisation du travail et tensions sociales : vers de nouvelles problématiques pour la GRH ? »

Commeiras N., Fournier C. et Loubès A., «Les tensions de rôle, la surcharge de travail et le système de contrôle : des déterminants de l'opportunité d'innovation dans le travail ? Une étude empirique auprès des managers de rayon »

Edey-Gamassou C., « Innover sans stress et gérer le stress en innovant : deux enjeux de gestion des ressources humaines »

Hechiche-Salah L., Bousnina-Bouallegue Z. et Touzani M., « La gestion du stress des enseignants à l'université : le cas de la Tunisie »

Steiler D. et Rüling C.-C., « Fusions, stress et stratégies d'ajustement : vers une perspective de recherche et d'innovation »

\section{Actes du Congrès de l'AGRH, 2004, Montréal}

Abord de Chatillon E., « Management de la santé et de la sécurité au travail : un problème de mesure ?»

Bachelard O. et Paturel D., « Nouvelles formes de souffrance au travail : analyses et réflexions en matière de GRH »

Cadieux, J., Roy, M., \& Desmarais, L. « Changement de perspective dans la mesure de performance en SST »

Carpentier S., « La remise en cause d'un système de gestion contribue-t-elle à la violence en entreprise ? Eléments de réponses par l'analyse du cas de commerciaux d'une PMI française. »

Dumas M., Alis D. et Campoy E., «L'apport des méthodes d'équations structurelles dans les études longitudinales d'analyse du changement attitudinal. Le cas de l'implication organisationnelle affective et de la perception du soutien organisationnel dans un contexte de changement du temps de travail»

Foucher R., Morin L., Bentein K. et Trottier M., «Mesurer l'engagement : l'intérêt de combiner les indices d'engagement affectif et normatif»

Guerrero S., « Proposition d'un instrument de mesure du contrat psychologique : le PCI »

Herrbach O. et Lérat-Pytlak J., « Implication et émotions au travail : une étude empirique »

Lapalme M.-E. et Doucet O., « Les récents développements dans l'étude de l'engagement des employés : la redondance perdure »

Mignonac K., «Que mesure-t-on réellement lorsque l'on invoque le concept de satisfaction au travail ? »

Poirot M., «L'expérience et les résultats de la mesure du stress dans six grandes entreprises françaises »

Quenneville N., Simard G. et Rabouin N., "L'influence de l'engagement affectif dans la relation entre la perception des pratiques de gestion des ressources humaines innovatrices et les comportements de mobilisation »

Saba T. et Lemire L. « Statut d'emploi et comportements au travail : l'effet de la violation du contrat psychologique »

Van Hoorebeke D., « Mesure de la dissonance émotionnelle au travail : un problème ethnométhodologique »

\section{Actes du Congrès de l'AGRH, 2005, Paris}

Abord de Chatillon E. et Bachelard O., « Je pollue, tu payes : un usage bien particulier du principe « pollueur payeur » dans le champ de la SST »

Alis D. et Dumas M., « 35 heures, conciliation vie familiale - vie professionnelle : le cas d'une conciliation inégalitaire »

Arnaud G., «De l'utilisation de la psychanalyse en sciences de la GRH : du traitement des objections à une possible convergence épistémologique»

Arnaud G. et Guinchard R., « Accompagnement et clinique du travail : propositions pour un coaching d'inspiration psychanalytique »

Barel Y. et Frémeaux S., « Perceptions par les salariés des mesures d'aide à la conciliation travail - hors-travail »

Benraïss L. et Meyssonnier R., «Proposition d'une échelle de mesure multidimensionnelle de la confiance du salarié à l'égard de son entreprise : vers la réconciliation entre l'économique et le social ?»

Campoy E. et Neveu V., « Améliorer la confiance pour améliorer la relation d'emploi »

Chanut-Guieu C., «Entre contraintes économiques et objectifs sociaux : l'examen de la satisfaction des dirigeants, bénévoles et salariés associatifs dans un contexte de changement»

Chênevert D., Charest A. et Simard G., «L'impact des modifications des pratiques et des politiques de GRH sur l'engagement affectif des employés »

Colle R., «L'influence des temps de travail personnalisés sur le bien-être des salariés »

De Bry F. et Galindo G., «L'organisation, nœuds de contrats psychologiques entre les parties prenantes. L'exemple des entreprises de biotechnologie»

Dolan S. L., Tzafrir S. S. et Baruch Y., « Testing the causal relationships between procedural justice, trust and organizational citizenship behavior» 
Edey-Gamassou C., «Épuisement professionnel et implication : un modèle fondé sur les ressources »

El Akremi A. et Sassi N., « Stress et violence organisationnelle : analyse d'une interaction « pathogène » en milieu professionnel » Guerrero S., Herrbach O. et Mignonac K., «L'engagement organisationnel des jeunes cadres : une question de soutien ou d'image »

Henrique Rogues de Souza P. et Iochins Grisci C., «Compression du temps dans les routines de production : souffrance psychique des journalistes du Sud du Brésil »

Hollet S., «Une validation de l'échelle d'engagement au travail (UWES - Utrecht Work Engagement Scale) auprès d'une population de commerciaux : l'antithèse positive de l'épuisement professionnel »

Moisson V. et Roques O., « La responsabilité sociale face au stress professionnel »

Naulleau M., « Rupture ou continuité des objets et dimensions de l'implication des salariés ayant vécu une période de restructuration? Intérêt exploratoire d'une perspective psychanalytique»

Normand N. et Tremblay D.-G., «Conciliation emploi - famille et intégration professionnelle : le cas de femmes professionnelles immigrées au Québec»

Ollier-Malaterre A., « Gérer le hors-travail ? Le cas des programmes Work/Life des entreprises américaines »

Piccinini V. C., Rocha de Oliveira S., Dos Santos Fontoura D. et Schweig C., « Quand travailler aurait-il un sens ?»

Poirot M., «Les compétences politiques et sociales comme remède aux stress des dirigeants ?»

Rocha de Oliveira S. et Piccinini V. C., « « Je travaille pour ton sourire ! » les sens du travail pour les dentistes filiés à l’UNIODONTO »

Tremblay M., Cloutier J. et Chênevert D., «Les pratiques et la performance prescrite et hors rôle : vérification du rôle médiateur de la justice, du support, de la confiance et de l'engagement organisationnel»

\section{Actes du Congrès de l'AGRH, 2006, Reims}

Arnaud G. et Guinchard R., « Existe-t-il un « désir de travail » ? Une réflexion exploratoire d'inspiration psychanalytique »

Asquin A., Garel G. et Picq T., « Quand les individus et les collectifs sociaux sont mis en danger par le travail en projet »

Banville B. et Chênevert D., «Les déterminants de l'absentéisme en milieu hospitalier : le rôle de l'engagement affectif et de l'épuisement professionnel»

Barel Y. et Frémeaux S., « Projets professionnels et aide à la conciliation vie professionnelle - vie personnelle »

Benedetto-Meyer M. et Illyine L., « Quand les facteurs de stress au travail sont d'ordre organisationnel... Quels outils d'analyse ? Quelles actions correctives?»

Boitel L., Castel M.-J., Delgoulet C., Jabot F., Juillard G., Presseq P., «Reconnaissance au travail et santé après 50 ans : une approche épidémiologique»

Bonnet M., Savall H. et Zardet V., « Lien entre GRH et contrôle de gestion dans le domaine de la santé au travail. Exemple d'une recherche - intervention centrée sur la prévention des troubles musculo-squelettiques»

Bouville G., « Absentéisme, âge et manque d'implication au travail : l'exemple des éboueurs »

Bouzidi S. et El Akremi A., « La reconnaissance au travail : étude d'un mode de construction identitaire »

Calvez V., « Construction du sens du travail et efficacité de l'organisation « déviante » : de la responsabilité comme compétence »

Carbonnel A., «Évolution de la perception des caractéristiques du travail et engagement dans le travail »

Castaing S. et Roussel P., «L'effet de l'évaluation du contrat psychologique sur l'implication affective dans la fonction publique : le rôle modérateur de la motivation à l'égard du service public»

Champion I. et Paturel D., « Harcèlement moral et risque organisationnel : un défi pour la santé au travail »

Chapon E., «Prévention de la violence: pour une gestion des ressources humaines des établissements scolaires. Cas de recherche intervention dans les lycées professionnels »

Chrétien L. et Létourneau I., « La gestion du travail en contexte de conciliation travail - famille »

Cintas C., «Un regard de GRH sur la violence au travail : le cas d'un hôpital psychiatrique »

Clergeau C., Detchessahar M., Devigne M., Dumond J.-P., Honoré L. et Journé B., « Transformations des organisations et santé des salariés : proposition d'un programme de recherche »

Colle R., Frimousse S. et Peretti J.-M., « Le défi de l'articulation vie privée - vie professionnelle des couples à double carrière »

Demery-Lebrun M., «Quelle opérationnalisation de l'implication pour concilier théories de la motivation et théories de l'implication? Réflexion en faveur d'une nouvelle échelle de mesure de l'implication dans le métier »

Doucet O., Simard G. et Tremblay M., «L'effet médiateur du support et de la confiance dans la relation entre le leadership et l'engagement»

Douillet P. et Sahler B., « La prévention des risques psychosociaux au travail : un défi stratégique pour les DRH » 
Douillet P., Fauconnier D., Pepin M. et Schweitzer J.-M., « Des coûts aux risques stratégiques : essai d'approche économique de la problématique des troubles musculo-squelettiques »

Dumas M., « Conflit et enrichissement travail et famille et implication : une étude des relations entre ces construits »

Dumond J.-P. « L'analyse longitudinale de l'absentéisme : considérations méthodologiques »

Duyck J.-Y. et Lahmouz K., «Implication organisationnelle et restructuration - L'effet modérateur de l'efficacité perçue : quelques réflexions à partir d'un cas dans le domaine des assurances »

El Akremi A., Haddaji N. et Sassi N., «Étude de l'impact des stresseurs organisationnels sur le bien-être et les comportements prosociaux des salariés dans un contexte de flexibilité »

Gambin R. et Lapeyrière S., « «Savoir parler du travail» : une compétence transversale, méconnue, perdue ? Et pourtant indispensable dans les mutations du travail»

Garreau L., « Création de sens et apprentissage organisationnel, une perspective croisée »

Guénette A.-M., Lee Y.-T, Papart J.-P., « Conditions de travail et santé : le modèle de Karasek revisité au travers d'une approche par les régressions polynomiales »

Gutiérrez-Martinez I., «Étude des dimensions affective et calculée de la double implication des professionnels de technologies d'information »

Hollet S., «Quels facteurs influencent l'engagement au travail ? Résultats d'une étude menée auprès de commerciaux »

Hubault F., « Le travail, un défi pour la GRH »

Lapalme M.-E., Simard G. et Tremblay M., « L'impact de la perception de rupture du contrat psychologique chez les travailleurs d'agence : une perspective de relation à multiples mandats »

Le Rendu-Lizee C., « La question du sens au cœur du changement organisationnel»

Leclercq E. et Potocki Malicet D., « Identités professionnelles et métiers des chercheurs »

Neveu J.-P., «Quand le cœur n'y est plus : de l'épuisement professionnel à l'agressivité envers le client »

Nkakleu R., " La tontine d'entreprise en tant que communauté de pratique, levier d'implication en situation interculturelle : une étude de cas longitudinale »

Poilpot-Rocaboy G. et Winter R., « Combattre le harcèlement psychologique au travail : proposition d'un processus d'intervention »

Suquet J.-B., «La GRH et la représentation du travail, entre activité subjective et activité prescrite: pour une prise en compte des dynamiques professionnelles »

Taktak Kallel I., «L'engagement des employés dans les centres d'appel en Tunisie : cibles, déterminants et conséquences sur l'efficacité au travail »

Tremblay D.-G., Najem E. et Paquet R., «Articulation emploi - famille et temps de travail : la présence d'enfants ou le sexe comme variable déterminante?

\section{Actes du Congrès de l'AGRH, 2007, Fribourg}

Abord de Chatillon E. et Desmarais C., « Pourquoi les encadrants sont-ils contents de travailler 54 heures par semaine ?»

Bouville G., « Les modèles explicatifs de l'absentéisme : question de mode ? »

Clergeau C., Geffroy-Maronnat B. et Pihel L., « Les déterminants organisationnels et managériaux de la santé au travail. Étude exploratoire au sein d'une PME »

Colle R. et Moisson V., «L'impact du stress professionnel et du déséquilibre effort/récompense sur l'intention de départ des infirmières »

Commeiras N., Loubès A., Fournier C., « Les managers face aux tensions de rôles : quelles incidences sur l'implication au travail ? »

Demery-Lebrun M., « La mesure de l'implication professionnelle : un exemple de corpus en évolution »

Galois I., « Soutien, confiance, satisfaction et engagement organisationnel affectif : mesure et relations entre les variables »

Guerrero S. et Herrbach O., « La confiance organisationnelle au cœur de l'échange social : et si bien traiter ses employés était payant ? »

Gutiérrez-Martinez I., «L'influence des pratiques de gestion de ressources humaines sur l'implication organisationnelle et professionnelle »

Peretti J.-P., Swalhi A., « Mesure de la fidélité organisationnelle »

Ruiller C., «Construction d'une échelle de la perception du soutien social : premiers résultats d'une étude de cas sur un centre hospitalier »

Stimec A., Bertrand T., Michel X. et Detchessahar M., «Contribution à la compréhension des facteurs organisationnels et managériaux de la santé au travail : le cas d'une usine d'un équipementier automobile » 
Amara M. Z. et Bietry F., « Le développement de l'implication organisationnelle par la confiance : application au contexte tunisien »

Clergeau C., Geffroy-Maronnat B. et Pihel L., «De la confiance à la distance, quand la GRH s'éloigne. Analyse des enjeux des transformations des choix de gestion des ressources humaines sur la relation à l'entreprise et la santé des personnels »

Colle R. et Christin J., « Couples à double carrière et articulation vie privée - vie professionnelle : le mariage de la carpe et du lapin ?»

Detchessahar M., Devigne M. et Stimec A., «Les modes de régulation du travail et leurs impacts sur la santé des salariés: deux établissements d'accueil des personnes âgées en quête de management»

Honoré L., « Management et santé au travail : quels liens et quels enjeux. Réflexions à partir de l'analyse du cas des ateliers de cueille de l'entreprise France Champignons »

Kouabenan D. R., « Rôle des croyances dans le management de la sécurité »

Lahmouz K. et Duyck J.-Y., «Le stress professionnel, l'implication organisationnelle et l'auto-efficacité : cas d'une restructuration - les apports d'une étude quantitative»

Marcq J., «L'influence du contexte de travail sur l'engagement des salariés »

Moisson V. et Edey-Gamassou C., "Stress, fatigue et soutien social : premiers résultats de deux études menées auprès du personnel soignant »

Ndao A. et Diop B., « Les causes de l'absentéisme dans les entreprises sénégalaises : le cas des Industries Chimiques du Sénégal (ICS) »

Ruiller C., «L'émotion et l'estime dans la construction de la perception du soutien social au travail : développement d'une échelle de mesure "

Sanséau P.-Y. et Smith M., « Work life balance in the context of regulatory change : a comparison of France and the UK »

\section{Actes du Congrès de l'AGRH, 2009, Toulouse}

Abord de Chatillon E. et Neveu J.-P., « Déviance organisationnelle et burnout : le cas d'une entreprise industrielle »

Alves S., Gosse B., Sprimont P.-A., « Test d'un modèle de déterminants et de conséquences de la satisfaction des salariés apprentis du supérieur »

Carpentier S. et Bachelard O., «La co-production de nouvelles pratiques en management de la santé et de la sécurité au travail: interrogation sur l'usage de différents types de méthodologies de recherche »

Detchessahar M. et Grevin A., «Le «tournant gestionnaire » des établissements de santé et son impact sur la santé au travail : le cas d'un centre de soins de suite malade de « gestionnite » »

Falcoz C. et Becuwe A., « Hétérosexisme, satisfaction et engagement des salarié-e-s gays et lesbiennes : études de variables explicatives et construction d'une échelle de mesure»

Gutiérrez-Martinez I., «Les intentions de turnover des professionnels du secteur des technologies d'information : le rôle médiateur de l'implication organisationnelle affective »

Poilpot-Rocaboy G., Notelaers G. et Hauge L. J., «Exposition au harcèlement psychologique au travail : impact sur la satisfaction au travail, l'implication organisationnelle et l'intention de départ »

Roques O.et Serrano Archimi C., «La perception du cynisme dans l'organisation : duplicité de rôle, stratégies d'ajustement et stress »

Tremblay M., Dahan J. et Gianecchini M., «Le succès en carrière et la satisfaction : étude de l'influence des critères de promotion et des ancres de carrière auprès d'une population d'ingénieurs "

Wuidar D., Stinglhamber F. et Hanin D., «L'impact du support organisationnel perçu et de l'interaction travail - famille sur le stress des policiers »

\section{Actes du Congrès de l'AGRH, 2010, Saint-Malo}

Abord de Chatillon E., Carrier Vernhet A. et Desmarais C., «Peut-on comprendre la souffrance psychosociale des salariés sans qu’ils ne s'en rendent compte?»

Alis D., « Le travail émotionnel des salariés en contact avec le public : prévenir les risques de dissonance »

Alves S., Gosse B. et Sprimont P.-A., « La satisfaction au travail des apprentis de l'enseignement supérieur : dimensions et conséquences »

Benion A., "Quelles conditions organisationnelles, managériales et de GRH pour l'émergence d'un environnement favorable à la construction de la santé des agents en centres d'appels?»

Besseyre des Horts C.-H. et Nguyen V., « Satisfaction, implication, engagement, enracinement et intention de départ des jeunes cadres : une relation ambiguë »

Bouville G., « La progression de l'absentéisme : nouveaux comportements des salariés ou nouvelles contraintes organisationnelles ? »

Carpentier S., « Nouveaux comportements et nouvelles pratiques managériales dans la grande distribution: le cas des managers de proximité face au management de la santé et sécurité au travail »

Clergeau C. et Pihel L., « Déterminants organisationnels et managériaux de la santé au travail : le rôle clé des managers de proximité dans 


\begin{tabular}{|c|}
\hline les centres d'appels» \\
\hline Codo S., « Engagement et stress au travail chez des managers territoriaux : quelles implications managériales ?» \\
\hline $\begin{array}{l}\text { Deme S., «Etude exploratoire des déterminants organisationnels et extraprofessionnels et des conséquences du conflit travail-famille : le } \\
\text { cas de trois femmes cadres avec enfants en bas âge. » }\end{array}$ \\
\hline $\begin{array}{l}\text { Desmarais C. et Dubouloy M., « Une double grille d'analyse pour évaluer les situations de cadres en difficulté : du contrat psychologique } \\
\text { au contrat narcissique» }\end{array}$ \\
\hline $\begin{array}{l}\text { Hollet-Haudebert S., Edey-Gamassou C. et Allard-Poesi F., « De l'instrumentation à l'instrumentalisation de la mesure du stress : analyse } \\
\text { du rapport d'enquête Technologia sur France Télécom » }\end{array}$ \\
\hline $\begin{array}{l}\text { Iglesias K., Renaud O. et Tschan F., « Satisfaction au travail : conséquences du choix des outils statistiques et des instruments de mesure en } \\
\text { GRH » }\end{array}$ \\
\hline $\begin{array}{l}\text { Ilama I. I., Belghiti-Mahut S. et Briole A., «Qualité de l'emploi, enjeux de la GRH dans l'aide à domicile : quelques indications d'une } \\
\text { étude exploratoire» }\end{array}$ \\
\hline Kilic S., « Quelles pratiques d'harmonisation vie privée - vie professionnelle pour quelles attentes des salariés ? » \\
\hline Moisson M., Fuhrer C. et Cucchi A., « Les déterminants du stress et de l'épuisement professionnel : le rôle de l'environnement relationnel » \\
\hline Molines M., « Leadership transformationnel, stress et performance : une étude multi niveaux » \\
\hline $\begin{array}{l}\text { Nkakleu R. et Manga B., «La tontine d'entreprise en tant que levier d'implication organisationnelle : résultats d'une étude auprès de } 225 \\
\text { cadres de PME camerounaises » }\end{array}$ \\
\hline Payre S., « Manager les hommes et leurs comportements afin de mieux prendre en charge les problèmes de santé au travail» \\
\hline Pezé S., « Les représentations du stress des dirigeants : quelles implications pour la gestion du stress au travail ? » \\
\hline Stimec A. et Michel X., « Santé au travail et performance durable : rapport au risque et mode de communication » \\
\hline Terramorsi P. et Peretti J.-M., « Le sentiment de reconnaissance au travail : proposition d'un instrument de mesure » \\
\hline Tremblay D.-G., « Articulation emploi - famille : la présence d’enfants est-elle déterminante pour le soutien organisationnel ? » \\
\hline Actes du Congrès de l'AGRH, 2011, Marrakech \\
\hline Abord de Chatillon E. et Desmarais C., « Le nouveau Management Public est-il pathogène ? » \\
\hline $\begin{array}{l}\text { Allard-Poesi F., Edey-Gamassou C. et Hollet-Haudebert S., « La construction du sujet souffrant au travail au travers des instruments de } \\
\text { mesure » }\end{array}$ \\
\hline $\begin{array}{l}\text { Ballesteros Levya F., Poilpot-Rocaboy G. et St-Onge S., « Les déterminants de l'articulation de la vie au travail et de la vie hors-travail des } \\
\text { travailleurs en mobilité : proposition d'un modèle de recherche» }\end{array}$ \\
\hline Bouville G. et Campoy E., « Les déterminants organisationnels d’une GRH « anti-bienveillante » : le cas du harcèlement moral» \\
\hline $\begin{array}{l}\text { Carrière J. et Bremner N., « Les effets des caractéristiques du travail sur l'épuisement professionnel dans un contexte hospitalier et l'effet } \\
\text { médiateur du sens du travail » }\end{array}$ \\
\hline $\begin{array}{l}\text { Chakor T., « La démarche de prévention des risques psychosociaux par les consultants externes : une approche en termes de représentations } \\
\text { sociales » }\end{array}$ \\
\hline $\begin{array}{l}\text { Chaudat P., "Animal de compagnie et facteurs d'implication et de réduction du stress dans l'organisation: quelles relations? Une } \\
\text { recherche sur la perception des salariés » }\end{array}$ \\
\hline $\begin{array}{l}\text { Chênevert D. Vandenberghe } \mathrm{C} \text { et Tremblay M., "Le rôle respectif des différents réseaux d'échanges sociaux associés aux conditions } \\
\text { psychologiques à l'égard de l'épuisement émotionnel et de la performance extra-rôle }: \text { le cas du personnel soignant » }\end{array}$ \\
\hline Cintas C. et Sprimont P.-A., « Soutien social et violence au travail : quels effets sur le burnout ? » \\
\hline $\begin{array}{l}\text { Closon C. et Leys C., « Relation entre les perceptions de la responsabilité sociale et l'implication affective et la satisfaction au travail. Le } \\
\text { rôle modérateur des attentes » }\end{array}$ \\
\hline Codo S., «Inégalités des managers publics territoriaux face au stress professionnel : impact des tensions de rôle » \\
\hline $\begin{array}{l}\text { Colin T. et Grasser B., « La prise en compte des aspects organisationnels dans le « bien-être » au travail : le cas des personnels d'un conseil } \\
\text { général » }\end{array}$ \\
\hline Conjard P., « Prévenir les risques psychosociaux par le management du travail» \\
\hline Duport M. et Janicot L., « Pour une GRH bienveillante et harmonieuse : santé, sécurité et RSE dans les entreprises en Chine » \\
\hline Fall A., « Pratiques de reconnaissance et motivation autonome des salariés : le cas d'une entreprise touristique française » \\
\hline $\begin{array}{l}\text { Garcias F., Dalmasso C. et Sardas J.-C., «Les déterminants socio-psychologiques de la santé au travail : modèles d'analyse et action sur } \\
\text { l'organisation » }\end{array}$ \\
\hline Garreau L. et Perrot S., « Le rôle des émotions dans la socialisation organisationnelle : une approche par le sen \\
\hline 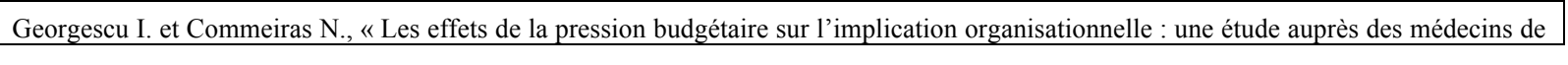 \\
\hline
\end{tabular}




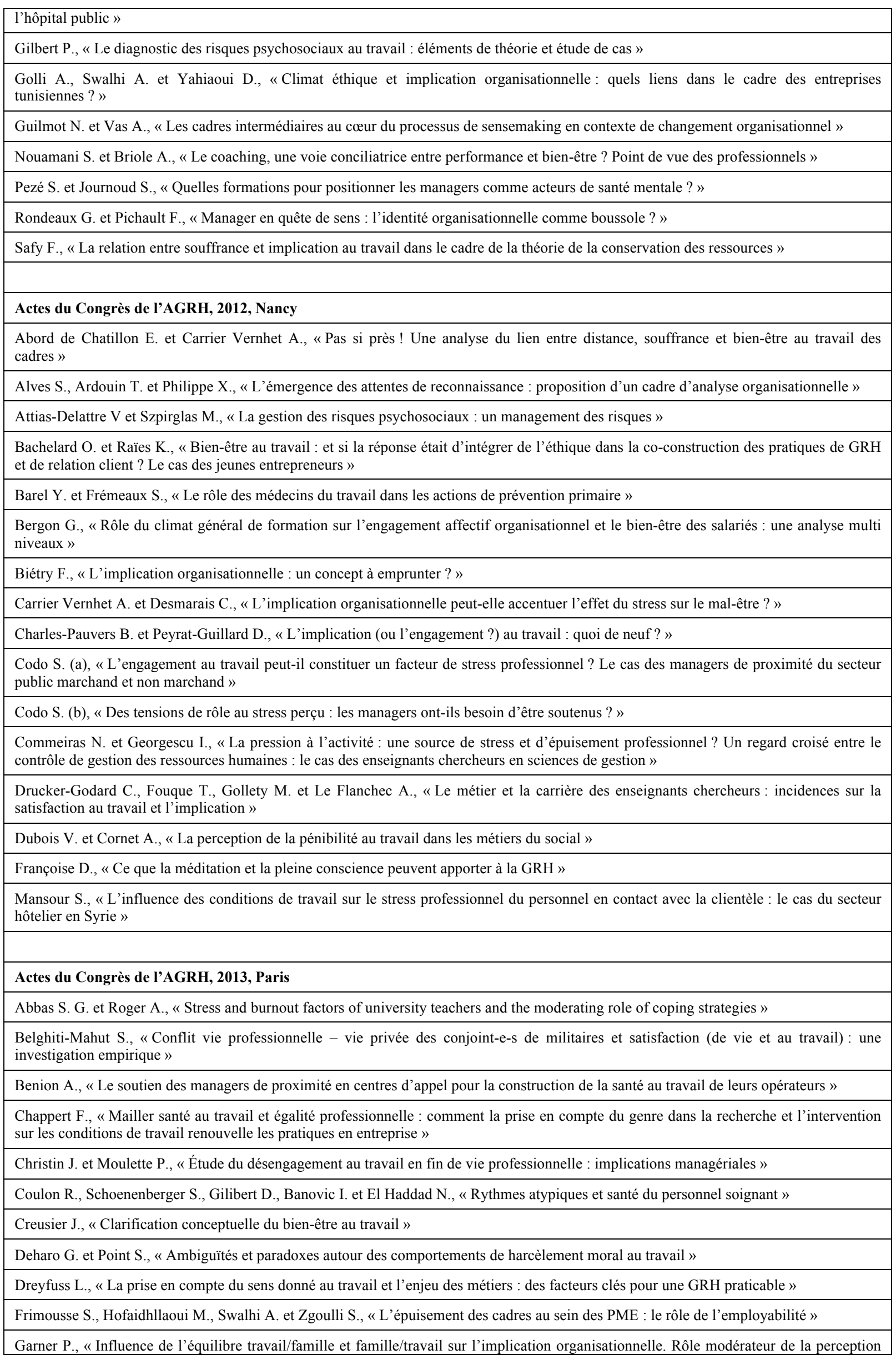




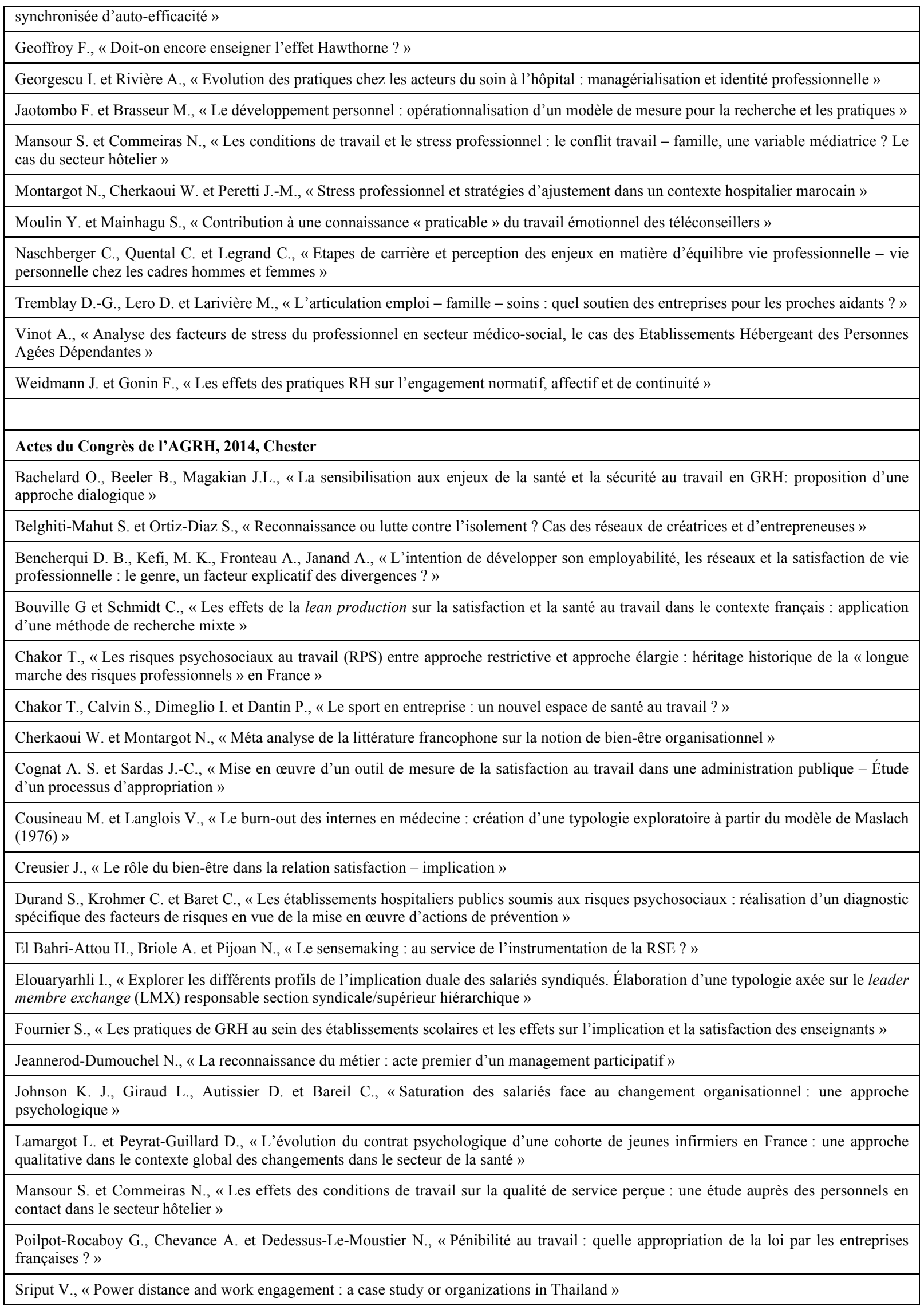




\section{Bibliographie complémentaire}

Abord de Chatillon, E., Bachelard, O., et Carpentier, S. (2012). Risques psychosociaux, santé et sécurité au travail: une perspective managériale, Paris: Vuibert, Collection recherche AGRH.

Abord de Chatillon, E., et Bachelard, O. (2005). Management de la santé au travail, Paris: L'Harmattan, Collection Conception et Dynamique des Organisations.

Autier, F., Ramboatiana, S. (2014). Travailler pour quoi faire? Le Mans: Gereso.

Chakor, T. (2014). « Les consultants dans la prévention des risques psychosociaux au travail : proposition d'une typologie de pratiques ». @GRH, 2014/1, n 10,37-58.

Chakor, T. (2015). "Généalogie des risques psychosociaux au travail : un phénomène au cœur d'une tension politique». Économies et Sociétés, Série «Études critiques en Management », $\mathrm{KC}, \mathrm{n}^{\circ} 4$, 2/2015, 197-225.

Gollac, M., Bodier, M. (2011). Mesurer les facteurs psychosociaux de risque au travail pour les maîtriser. Rapport du Collège d'expertise sur le suivi des risques psychosociaux au travail, faisant suite à la demande du Ministre du Travail, de l'Emploi et de la Santé, Avril.

Grasset, Y., Debout, M., Rouat, S., \& Bachelard, O. (2008). Risques psychosociaux, vraies questions, bonnes réponses. Paris: Editions Liaisons.

Hirigoyen, M.F. (1998). Le harcèlement moral : la violence perverse au quotidien. Paris: La Découverte.

Lachmann, H., Larose, C., \& Penicaud, M. (2010). Rapport sur le bien-être et l'efficacité au travail. 10 propositions pour améliorer la santé psychologique au travail. Rapport fait à la demande du Premier ministre, Février.

Nasse, P., \& Légeron, P. (2008). Rapport sur la mesure et le suivi des risques psychosociaux au travail. Rapport au Ministre du Travail, des Relations sociales et de la solidarité, 12 Mars. 